\title{
Quantum Cosmology from Group Field Theory Condensates: a Review ${ }^{\star}$
}

\author{
Steffen GIELEN ${ }^{\dagger}$ and Lorenzo SINDONI ${ }^{\ddagger}$ \\ $\dagger$ Theoretical Physics, Blackett Laboratory, Imperial College London, London SW7 2AZ, UK \\ E-mail:s.gielen@imperial.ac.uk \\ ¥ Max Planck Institute for Gravitational Physics, Am Mühlenberg 1, 14476 Golm, Germany \\ E-mail: lorenzo.sindoni@gmail.com
}

Received February 29, 2016, in final form August 12, 2016; Published online August 18, 2016

http://dx.doi.org/10.3842/SIGMA.2016.082

\begin{abstract}
We give, in some detail, a critical overview over recent work towards deriving a cosmological phenomenology from the fundamental quantum dynamics of group field theory (GFT), based on the picture of a macroscopic universe as a "condensate" of a large number of quanta of geometry which are given by excitations of the GFT field over a "no-space" vacuum. We emphasise conceptual foundations, relations to other research programmes in GFT and the wider context of loop quantum gravity (LQG), and connections to the quantum physics of real Bose-Einstein condensates. We show how to extract an effective dynamics for GFT condensates from the microscopic GFT physics, and how to compare it with predictions of more conventional quantum cosmology models, in particular loop quantum cosmology (LQC). No detailed familiarity with the GFT formalism is assumed.
\end{abstract}

Key words: group field theory; quantum cosmology; loop quantum gravity

2010 Mathematics Subject Classification: 83C45; 83C27; 83F05; 81T27

\section{Introduction}

The idea that spacetime and geometry should be emergent, i.e., an effective description of the collective behaviour of different, "pre-geometric" fundamental degrees of freedom, is now a common theme in most approaches to quantum gravity [104]. In particular, in theories formulated fundamentally in terms of discrete, combinatorial structures rather than continuum variables (such as a spacetime metric or connection on a smooth manifold) in order to avoid the problem of perturbative non-renormalisability of general relativity [76], the emergent nature of a continuum description is a necessity, in order to connect to other areas of physics and to our experience which take place in the continuum. Showing how a continuum emerges is hence the challenge faced by loop quantum gravity $[8,124,131]$ and spin foam models [117, 125], causal dynamical triangulations [4], causal set theory [82], as well as tensor models [122] and group field theories [18, 91, 103], a challenge which has been addressed with varying success. A separate notion of emergence of dynamical spacetime arises in the AdS/CFT correspondence [95] for supersymmetric theories, which posits that a continuum quantum spacetime geometry is in a precise sense dual to a lower-dimensional conformal field theory on a fixed geometry. These rather different notions of emergence all face the challenges of (nonlocally [98]) mapping the fundamental degrees of freedom to the sought-after emergent spacetime description.

In a group field theory (GFT), spacetime emerges from combinatorial structures, labelled by additional geometric or pre-geometric data. In the simplest representation of simple GFT

\footnotetext{
${ }^{\star}$ This paper is a contribution to the Special Issue on Tensor Models, Formalism and Applications. The full collection is available at http://www.emis.de/journals/SIGMA/Tensor_Models.html
} 
models, this data consists of group elements attached to the edges of a graph, typically of fixed valency. The group elements themselves are interpreted as parallel transports of a gravitational connection along these edges, and hence are analogous to the variables of lattice gauge theory. Unlike in lattice gauge theory, the graph or lattice does not carry a clear physical interpretation: graphs appear as Feynman diagrams in the perturbative expansion around a GFT vacuum, and quantities of interest such as the partition function contain a summation over an infinite number of such graphs. In general, there is little reason to trust perturbation theory, i.e., a truncation of this sum to a few simple graphs, since there is no small expansion parameter and no interpretation of the vacuum as a physically meaningful geometry. Indeed, viewing GFT as a second quantisation formulation of loop quantum gravity (LQG) and hence adopting operators for geometric observables such as volumes and areas from LQG [105], the most natural GFT vacuum is a state in which expectation values for such geometric observables vanish - it is a 'nospace' state, analogous to the Ashtekar-Lewandowski vacuum [6] of LQG. An approximately smooth, macroscopic geometry of physical interest can hardly be found as a small perturbation of such a vacuum.

Since the early days of LQG, some effort has been invested into the construction of states that can approximate continuum geometries, such as flat space [10]. These necessarily excite a large number of degrees of freedom compared to the Ashtekar-Lewandowski vacuum, and accordingly are described by a large graph with many edges and vertices. One would hope to find states that can play the role of a new vacuum of the theory around which a different notion of perturbation theory can be defined, e.g., in order to compare LQG with the perturbative quantisation programme of continuum general relativity. Completing this task requires knowledge about the dynamics of many interacting degrees of freedom, which has been difficult to extract from traditional LQG and its spin foam formulation, partly due to the rather complicated combinatorial structure of existing models. In the study of spin foam models such as the currently favoured EPRL model [62], known results for transition amplitudes are mainly restricted to asymptotic regimes, in particular in the "large- $j$ limit", see, e.g., [22, 81].

The situation calls for the development of systematic methods to treat many degrees of freedom in LQG, beyond the truncation given by a fixed graph. One possibility is to adopt methods from lattice gauge theory and use coarse graining or renormalisation procedures in order to obtain solutions to the dynamics and ultimately a theory with a well-defined continuum limit, where diffeomorphism symmetry is restored [54]. The endpoint of such a renormalisation group flow corresponds to a particular continuum phase, describing the collective behaviour of many degrees of freedom (infinitely many in the limit). In an interpretation of the setting as a theory of quantum gravity, one would like to show that such a phase describes a smooth non-degenerate spacetime geometry, such as Minkowski or de Sitter space.

In the GFT setting, one can also explore a different route. As a second quantisation formulation of LQG, the main advantage of GFT is the availability of quantum field theory methods when treating many degrees of freedom in the language of quantum fields rather than $N$-particle states. Conceptually, the situation is rather similar to the use of (non-relativistic) quantum field theory in condensed matter physics, when one is interested in the collective behaviour of a large number of interacting atoms or molecules, rather than perturbative scattering around the Fock vacuum. One of the most relevant examples of the successful application of quantum field theory in condensed matter physics is the study of Bose-Einstein condensation and superfluidity [118]. In this context, the condensation of many atoms into a common ground state can be viewed as a transition from a perturbative phase around the Fock vacuum (of zero atoms) into a condensed phase, with associated symmetry breaking of the U(1) symmetry of the theory. Similarly, the language of GFT allows a treatment of many degrees of freedom through quantum fields rather than a fixed graph or " $N$-particle state". The elementary building block of a graph, a vertex of given valency, then plays the role of an atom of geometry. In fact, this interpretation is quite literally 
the one in LQG: the volume operator only has non-zero eigenvalues when acting on vertices [124, 131], so that such vertices do indeed form the "atoms of space". Using not a fixed number of such atoms but more general states that contain a superposition of many (typically infinitely many) graphs is quite natural in LQG where the (kinematical) Hilbert space is a direct sum of Hilbert spaces associated to different graphs $[124,131]$. The continuum limit of an infinite number of such atoms is the usual thermodynamic limit of statistical field theory. This view suggests that a very natural candidate for a configuration of many degrees of freedom with high coherence and symmetry, and potentially a candidate for a non-perturbative vacuum of LQG, should be given by a group field theory condensate analogous to a Bose-Einstein condensate [101, 102] .

Given that this idea of a macroscopic spacetime as a kind of condensate of microscopic degrees of freedom is rather natural, it may not appear surprising that it has already been suggested in other contexts. In LQG, it was suggested by Koslowski [90] and shown in more detail by Sahlmann [127] that the Ashtekar-Lewandowski construction, leading to a unique vacuum in LQG [93], could be generalised to allow for a larger class of vacua, where expectation values for geometric operators are shifted by a given classical field configuration with respect to the Ashtekar-Lewandowski vacuum, so that one can choose for example a vacuum corresponding to continuum flat space. One can view the Koslowski-Sahlmann vacua as implementing a breaking of diffeomorphism symmetry which would require these expectation values to vanish. There has not yet been much work on understanding these vacua as a dynamically emerging configuration; excitations over them are also still discrete structures as in usual LQG.

There is also a more "bottom-up" motivation from quantum cosmology for thinking of a macroscopic universe as a sort of condensate [35]. In quantum cosmology, the traditional strategy since Misner [99] for obtaining a manageable quantum model of the universe has been to perform a "minisuperspace" reduction at the classical level, i.e., to assume that the universe is spatially homogeneous, and to quantise only the remaining degrees of freedom of geometry. The assumption of exact homogeneity means that one focuses on the local evolution of a small patch around a single point in space, as there are no spatial gradients by construction, and one imposes the symmetry-reduced quantum Einstein equations as the dynamics of such a patch. As explained in [35], this procedure can be thought of as a "single-patch" quantum model in which one quantises a single "atom" or chunk of space, and assumes that the dynamics of the atom is related to the effective dynamics at large scales. This view would suggest passing to a "many-patch model" of many interacting chunks of space, as a way to check the validity of this assumption, and to extend the model to include inhomogeneities. Depending on the length scales associated to these chunks, this idea can be related to the separate universe picture in cosmology [133], where inhomogeneities in a certain range of wavelengths can be included by modelling an inhomogeneous universe as composed of many small, locally homogeneous patches. In such a many-patch model, given that inhomogeneities are very small in the observed Universe, one then considers states with weak interactions between different patches. A simple ansatz for the many-patch wavefunction would be a product state, so that there is a collective single-particle wavefunction describing the state, again very similar to states used for (weakly interacting) Bose-Einstein condensates. This idea was explored in [37] in a lattice model starting from classical general relativity, where one finds that the existence of interactions between patches leads to a nonlinear and nonlocal evolution equation for the single-particle wavefunction, similar to the Gross-Pitaevskii equation in condensed matter physics [118]. We will find very similar qualitative features in the effective dynamics of GFT condensates.

The application of loop quantum gravity techniques to quantum cosmology led to the development of loop quantum cosmology (LQC) [11,32]. LQC combines "top-down" and "bottom-up" elements: while the classical Friedmann equation is used as a starting point for the dynamics, one also uses the kinematics of LQG, so that the continuum Ashtekar-Barbero connection [5, 19] is replaced by finite parallel transports along edges of a graph, and the Wheeler-DeWitt equation 
becomes a difference rather than a differential equation. An open issue that has attracted a lot of interest in recent years (see, e.g., [1, 30, 31, 61, 64] for various approaches) is how to embed the kinematics and dynamics of LQC into full LQG, or rather derive LQC as an approximation or effective description of the full theory of LQG when applied to almost exactly homogeneous, macroscopic Universes like our own. In this review, we explore another viewpoint in which one thinks of quantum cosmology as the effective dynamics of a condensate of microscopic degrees of freedom of quantum geometry. In short, "quantum cosmology is the hydrodynamics of quantum gravity". The condensate hypothesis provides methods for deriving an effective description of the dynamics of realistic universes in GFT, which can then be compared with conventional quantum cosmology models such as LQC, as well as ultimately with observation.

\subsection{Relation to analogue and emergent gravity}

The philosophy we are following shares some common ground with the philosophy behind the programmes of "analogue" and "emergent" gravity. Analogue models for gravity [21] seek to emulate some physical properties of gravitational systems, such as Hawking radiation [132], by non-gravitational systems usually described by some form of hydrodynamics. Motivated by the derivation of the Einstein equations from thermodynamics [86], and more generally the close link between spacetime geometry and thermodynamics, following Boltzmann's dictum "if you can heat it, it must have microstructure" [115], emergent models go one step further and aim to describe dynamical gravity as emerging from an effective description of different fundamental degrees of freedom [128]. The idea that spacetime is a condensate of fundamental "atoms of spacetime" has been suggested in this context of emergent gravity [84], without an obvious proposal for the nature of the "atoms".

Group field theories provide a natural candidate for a theory of the atoms of spacetime and their dynamics, and suggest that classical gravitational dynamics can be found in an effective hydrodynamic description of the dynamics of a GFT condensate, as suggested in the context of lower-dimensional models in [110]. The results and methods covered in this review show the detailed application of these ideas to cosmology.

\subsection{Relation to studies of renormalisation and phase structure in LQG/GFT}

The study of condensates in GFT can be seen as part of a larger effort aimed at understanding the collective dynamics of many interacting degrees of freedom of quantum geometry in loop quantum gravity and its generalisations, including the more general setting of GFT. Given the interpretation of GFT condensates as macroscopic continuum geometries, one would like to show that such condensates correspond to a dynamical phase of a known GFT model, at least in a certain range of the free parameters (coupling constants). One would also like to understand the possible phase transitions, in particular transitions between a "pre-geometric" and a "geometric" continuum phase such as described by a condensate. Replacing the classical Big Bang singularity by such a phase transition is the idea often dubbed geometrogenesis, and first advocated in the context of "quantum graphity" [97]. Showing that geometrogenesis can occur in the setting of GFT would mean a revolutionary reinterpretation of the origin of space and time, as it would show the emergence of a GFT phase with macroscopic geometric interpretation (in terms of a four-dimensional, continuum spacetime, causal structure, a metric, etc.) from a framework which is fundamentally formulated without any of these structures, and instead just uses combinatorial and group-theoretic data.

The main tool for mapping out the phase diagram for GFT and related theories is the renormalisation group, particularly its Wilsonian formulation based on effective actions. Applying renormalisation tools to GFT has been an extremely active field of research in recent years, 
see, e.g., [23, 27, 46, 47, 48]. A major result is that many models studied so far (of the tensorial GFT type) exhibit asymptotic freedom [23, 123], making them potential candidates for a fundamental theory. The infrared limit of the renormalisation flow is then important in order to understand the hypothesis that spacetime is a GFT condensate (of many "small" quanta). Here, in some models evidence has accumulated for phase transitions between a symmetric and a condensate-type phase $[25,26]$, just of the type we are interested in, with an associated flow to an interacting fixed point in the IR. So far, no such renormalisation analysis has been performed for GFT models with direct relation to LQG, such as the EPRL/FK GFT [24], but progress towards the study of more and more sophisticated models has been rapid. Ultimately, one would aim to justify the assumption of a GFT condensate in the models we are looking at from a more rigorous analysis of this type.

There has also been a lot of activity in the renormalisation analysis of simplified and generalised spin foam models by coarse graining methods, aimed at finding a continuum limit that restores diffeomorphism invariance $[53,56,57]$. Part of this effort has culminated in the development of a new representation for LQG $[13,55]$, with a new vacuum that is in some sense dual to the Ashtekar-Lewandowski vacuum of traditional LQG: it is a vacuum of exactly flat connections but completely undetermined conjugate metric variables. These results already show that phases of LQG and spin foam models beyond the degenerate phase around the Ashtekar-Lewandowski vacuum exist, giving further motivation to the study of GFT condensates, although the detailed connection between these research programmes still needs to be understood better.

\subsection{Structure of this review}

After giving some perspective in this introductory section, our aim is to give a self-contained overview over the research of roughly the last three years on condensates in GFT and their relation to quantum cosmology. To this end, in Section 2 we introduce the basics of the GFT formalism, set up notation for this review, and define the class of models we will be looking at. While some of this material will be known to readers familiar with the GFT literature, they might want to consult it for clarity on which GFT models we are studying and on notational conventions. In Section 3, we outline the (kinematical) definition of condensate states in GFT, mainly focusing on the simplest case that is in direct analogy with real Bose-Einstein condensates. Many conceptual and technical points regarding the interpretation of these condensates in terms of spacetime geometry will be explained. We also touch upon a more general class of condensate states introduced more recently. In Section 4, we show how to extract information about the dynamics of such condensates from the fundamental quantum GFT. We give different methods for extracting effective dynamical equations, explain the relation to quantum cosmology and the approximations and truncations involved. We also go into more detail as to how such effective dynamics can be compared with models such as LQC. In Section 5, we finally summarise the main results, open questions and future directions of this approach.

\section{Group field theory (GFT)}

In this section we will present the main ideas and basic technical tools of GFT that will be needed for the application to cosmology. The presentation will be self-contained; for further background on the general setting of GFT and recent progress, we refer to the reviews [18, 91, 103].

Group field theories are quantum (or statistical) field theories defined over group manifolds, most commonly restricted to classes of theories which can be designed to provide a possible definition of a path integral for quantum gravity. In a nutshell, we are interested in models for which the perturbative expansion of its partition function or field equation, and the perturbative 
expansion of the correlation functions, generates a sum over graphs that can be seen as dual to discretisations of manifolds.

In addition to the peculiar combinatorial structure, similar to that found in matrix and tensor models after which GFTs are modelled, the graphs of GFT are decorated by data related to the chosen group (group elements, representation labels or Lie algebra elements). The role of these additional data is to store geometric information (metric or connection data) beyond the mere combinatorial/topological structure. In fact, this group-theoretic data corresponds precisely to the basic variables of LQG, holonomies of a connection and fluxes of a triad field. In this way, GFT attempts to define a sum over discretised geometries which can be used, once a continuum limit is identified, to obtain a path integral for quantum gravity.

The definitions given so far cover a fairly rich class of models. In this review, we focus on simple models that have so far mainly been used in the application to cosmology, but the constructions of GFT condensate states have straightforward extensions to more complicated models. In particular, the desired property of the sum over graphs - that it represents a sum over combinatorial manifolds, or at least pseudomanifolds - is generally achieved by introducing coloured models with more than one fundamental GFT field [78, 80]. We will not be discussing coloured models in any detail, but it should be clear in the following that the main ideas are generally applicable to a wide class of GFT models.

\subsection{Definition of the basic models}

There are two prototypes of GFT that are useful for applications to quantum gravity, which both define a quantisation of topological BF theories over random manifolds. The first one is the Boulatov model [41], whose partition function can be naturally rewritten in terms of the Ponzano-Regge model [120] for three-dimensional (Riemannian) quantum gravity.

The second model, defined by Ooguri [100], can be seen as the four-dimensional counterpart of the Boulatov model. It generates a Feynman expansion which can be related to the amplitudes of a topological BF quantum field theory, the backbone of the spin foam approach to quantum gravity in four dimensions [117]. For the application to cosmology, we focus on four dimensions, so that we will be interested in models built on extending the Ooguri model from a topological theory to a theory of quantum gravity. We will comment later on various possible generalisations. We will also focus on the pure gravity case, as most models so far aim to define pure quantum gravity. The extension to matter coupling is of course crucial for a more realistic cosmology, and will be discussed in Section 4 .

The starting point is the choice of a Lie group $G$, which will select the type of data appearing in the Feynman diagrams, and will be interpreted as the local gauge group of gravity. Typical choices are $G=\mathrm{SU}(2), \mathrm{SO}(4)$, $\mathrm{Spin}(4)$ or $\mathrm{SL}(2, \mathbb{C})$. The second main choice is the combinatorial structure of the elementary building blocks used to build spacetime geometry. For four-dimensional spacetime and three-dimensional space, the simplest possibility is to use simplicial building blocks: the elementary building block of three-dimensional space is then a (quantum) tetrahedron [20]. In the dual picture familiar from LQG and spin foams, it corresponds to a central vertex with four open outgoing links, with group-theoretic data associated to these links. The field theory is accordingly defined on four copies of $G$ (see Fig. 1). With these choices, the elementary quanta of the theory can be assembled into three-dimensional simplicial geometries that represent the boundary states of the theory. This interpretation in terms of boundary configurations of four-dimensional geometries is then completed by the examination of the perturbative expansion of the correlation functions that they encode, once the dynamics with the correct combinatorial structure is introduced. It is the interplay between the combinatorics of the equations of motion and the number of group arguments of the fields, together with the gauge group used, that suggests the interpretation of the quanta and their interactions in 

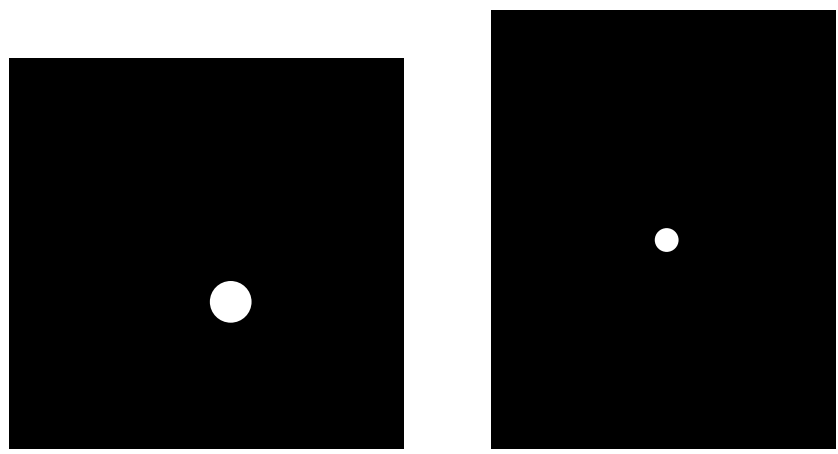

Figure 1. GFT quanta as open spin network vertices (left) or quantum tetrahedra (right).

geometrical terms. Indeed, with different dynamics one could also construct a field theory based on fields with four arguments which has a $2+1$-dimensional interpretation, with basic quanta interpreted in terms of quadrilaterals. Generalisations of GFT in which the restriction to simplicial geometry is dropped and general three-dimensional polyhedra appear as elementary quanta have been constructed [109]. These generalisations are motivated primarily by the appearance in canonical loop quantum gravity of graphs with vertices of arbitrary valency, corresponding to general polyhedra [28], where a restriction to tetrahedra seems arbitrary. Restricting to simplicial building blocks means one can keep track of the topology of the graphs transparently, and is certainly the simplest choice, on which we focus in the following.

Superficially, these choices seem to imply that we are assuming spacetime remains fourdimensional up to the very smallest scales, but this is not true: any useful notion of dimensionality would apply to the collective properties of a number of elementary quanta, rather than a single one. Experience from approaches to quantum gravity that fundamentally involve discrete structures shows that one should then expect a dimensional flow when going from macroscopic to microscopic scales, where the nature of this flow (and in particular, the question of whether four dimensions are reached at large scales) depends highly non-trivially on the details of the model, and not simply on the "dimensionality" used for the fundamental building blocks of the theory. For some results relevant in the GFT context, see [45].

After these basic choices, the field theory is specified by a choice of a type of field and by the corresponding action. The most common choices are real or complex scalar fields. While topological models can often be based on a real field, in order to set up the Fock space structure required for the discussion of GFT condensates, a complex field will be required. In either case, a simple general form of the action, for compact $G$, is

$$
S[\varphi]=\int \mathrm{d} g \mathrm{~d} g^{\prime} \bar{\varphi}\left(g_{I}\right) K\left(g, g^{\prime}\right) \varphi\left(g_{I}^{\prime}\right)+\frac{1}{5 !} \int(\mathrm{d} g)^{5} V\left(g^{1}, \ldots, g^{5}\right) \prod_{j=1}^{5} \varphi\left(g_{I}^{j}\right)+\text { c.c. },
$$

where here and in the following we are using a condensed notation. The arguments of the field are $g^{i} \equiv g_{I}^{i}=\left(g_{1}^{i}, g_{2}^{i}, g_{3}^{i}, g_{4}^{i}\right)$, and we are often suppressing the lower index running over the four group elements. The symbol $\int \mathrm{d} g$ stands for an integration over $G^{4}$, where $\mathrm{d} g$ is the Haar measure on $G^{4}$, normalised to unit total volume. Accordingly, $\int(\mathrm{d} g)^{5}$ means integration over $G^{4 \cdot 5}=G^{20}$. This notation will be useful, as one is almost always interested in $G^{4}$ or products of several $G^{4}$ domain spaces. In the few cases where a different integration domain is needed, it will be specified explicitly (see, e.g., (2.5)), so that no confusion should arise.

One also needs to specify the function space that is assumed to be integrated over in the GFT path integral. In the perturbative approach, we can assume that this space is the space of square-integrable functions on $G^{4}$. Since the interaction terms involve higher powers of the fields in convolution with variously constructed kernels, it is necessary to check that these terms 
are well-defined in the given space of functions and, if needed, to introduce a regularisation, as we will discuss in one example below (2.2).

The kernels $K\left(g, g^{\prime}\right) \equiv K\left(g_{I}, g_{I}^{\prime}\right)$ and $V\left(g^{1}, \ldots, g^{5}\right) \equiv V\left(g_{I}^{1}, \ldots, g_{I}^{5}\right)$ determine the details of the resulting Feynman amplitudes. The choice of an interaction term containing five copies of the field in (2.1) is suggested by the interpretation of these amplitudes in terms of four-dimensional flat simplices glued along common boundaries, i.e., sharing tetrahedra on their boundaries.

This action can be generalised in many different ways. One obvious possibility is to introduce more terms in the interaction potential, admitting an interpretation of simplicial polytopes (i.e., polytopes whose boundaries are made of simplices). For now, we focus on the well-understood simplicial case. More importantly, in the construction of models relevant for quantum gravity rather than topological field theory, one needs to include the so-called simplicity constraints into the interaction $V$; we will detail this in Section 2.5.

Apart from the motivation coming from the interpretation as simplicial geometry, one can also consider different possible interaction terms in (2.1) from a quantum field theory perspective, in particular by studying their renormalisability. Indeed, the construction of renormalisable and asymptotically free GFT models has been the focus of recent work, see Section 1.2.

An important property of the GFT field is a type of discrete gauge invariance, similar to lattice gauge theory: as our group elements will be interpreted in terms of parallel transports of a connection, from a vertex to the endpoint of an edge, we should make sure that only gaugeinvariant information is used. A simple choice is to restrict the field configurations to those which are diagonal under the right diagonal action of $G$ on $G^{4}$ :

$$
\varphi\left(g_{1} h, g_{2} h, g_{3} h, g_{4} h\right)=\varphi\left(g_{1}, g_{2}, g_{3}, g_{4}\right), \quad \forall h \in G .
$$

While this is the simplest option, there are other possibilities: for instance, one can use general fields on $G^{4}$ but include into the potential term a suitable projector that maps functions on $G^{4}$ onto functions on $G^{4} / G$. In the following we will keep using $G^{4}$ as the domain of the fields, even though we will impose gauge invariance directly on the field. While this means that the parametrisation is redundant, it greatly simplifies the construction of the models.

The symmetry (2.2) leads to some technical complications if one wants to extend the construction to non-compact $G$, in particular $G=\operatorname{SL}(2, \mathbb{C})$ as the Lorentz group in four dimensions. Indeed, as one can see easily, for $G=\mathrm{SL}(2, \mathbb{C})$ the action $(2.1)$ is ill-defined; because of the symmetry property $(2.2)$ of the field, there is a redundant integration over $\operatorname{SL}(2, \mathbb{C})$ with infinite volume. This issue can be fixed rather easily, either by working with a gauge-fixed field on $\mathrm{SL}(2, \mathbb{C})^{4} / \mathrm{SL}(2, \mathbb{C}) \simeq \mathrm{SL}(2, \mathbb{C})^{3}$, or by simply removing redundant integrations in the kinetic and interaction terms, keeping arguments of the fields fixed. We refer to Appendix A of [72] for a detailed discussion of these subtleties (and their resolution) for the Ooguri model, but for the purposes of this review just stress that they pose no substantial difficulty.

In order to finally be more concrete, choosing a real field $(\varphi=\bar{\varphi})$, the simple choices

$$
K\left(g, g^{\prime}\right)=\prod_{I} \delta\left(g_{I}^{\prime}\left(g_{I}\right)^{-1}\right)
$$

for the kinetic term and

$$
V\left(g^{1}, \ldots, g^{5}\right)=\lambda \prod_{e} \delta\left(g_{l(s(e))}^{s(e)}\left(g_{l(t(e))}^{t(e)}\right)^{-1}\right)
$$

for the interaction term lead to the Ooguri model. Here we are denoting by $e$ the ten edges of a complete graph of five vertices (see Fig. 2), by $s(e)$ the copy of the field associated to the source of the edge, by $t(e)$ the target, and by $l(s(e))$ (and $l(t(e)))$ the group element involved. This choice of combinatorics encodes the pattern of gluings in order to form a 4 -simplex out 


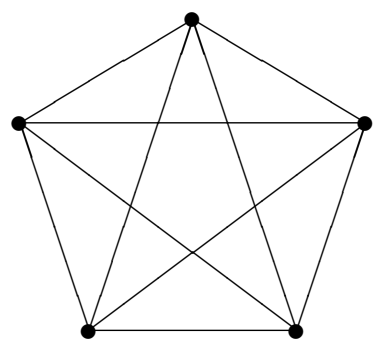

Figure 2. Complete graph of five vertices.

of five tetrahedra. As we shall see later, by modifying the kernels $K$ and $V$ it is possible to generate models that are related in a precise way to spin foam models [117].

The partition function obtained from these ingredients,

$$
Z=\int \mathcal{D} \varphi \exp (-S[\varphi])
$$

when treated as a perturbative expression in the GFT coupling constant $\lambda$, generates a sum over graphs that can be seen as dual to simplicial complexes. The amplitudes corresponding to the Ooguri model can be shown to be the amplitudes of a discretised BF theory [100],

$$
Z=\sum_{\Gamma} \lambda^{\# V(\Gamma)} \mathcal{A}(\Gamma)=\sum_{\Delta(\Gamma)} \lambda^{\# V(\Gamma)} \mathcal{A}_{B F}(\Delta(\Gamma))
$$

with a sum over the graphs $\Gamma$ being reinterpreted as a sum over discrete simplicial complexes $\Delta(\Gamma)$. It is precisely the possibility of generating a sum over (random) simplicial complexes that makes GFT relevant for the definition of a discrete path integral for the gravitational field. The choice of the Euclidean partition function assumes implicitly that, after its proper definition, it will be analytically continued, once a suitable parameter has been introduced, to the quantum-mechanical partition function. Since we will be working directly in the quantum operatorial version, this choice plays no role in the rest of the presentation.

The main problem that we are left with, then, is to make sense of formal expressions as this partition function. In this review, we mainly focus on the attempts at the extraction of their physical content which follow the ideas of condensed matter physics for many-body problems.

\subsection{Second quantisation, states associated to closed graphs, and operators}

Before discussing the modification of the topological kernel (2.3) necessary to formulate quantum gravity models and not just topological field theories, we briefly present GFT from the canonical viewpoint of field operators. While the operatorial version of the models does not add anything substantially new to the general idea of obtaining spacetime from Feynman diagrams, it is the key ingredient that will allow us to make significant progress in the calculation of physical predictions. Indeed, the language of Fock spaces and field operators acting on them will allow us to attack the problem of understanding the physical implications of GFT using a number of powerful techniques adapted from condensed matter physics. Thanks to this reformulation, we will be able to design quantum states with clearer geometric interpretation, which can include some educated guesses about possible nontrivial nonperturbative aspects of the theory in a simple and controlled manner. Additionally, the connections (and differences) to canonical LQG can be stated more precisely [105], without going through the spin foam formalism.

The canonical formalism has clear advantages. Besides the construction of interesting quantum states, in order to answer specific physical questions we need to understand how to compute 
the expectation values of physical quantities. The second quantised formulation that we are going to use will allow us to translate and generalise geometric operators introduced in LQG.

The construction follows the standard construction of nonrelativistic quantum field theories. It is important to stress, however, that we are not viewing it as originating from the canonical quantisation of a field theory: there is no canonical choice of "time variable" on the domain space of GFT that would allow a definition of generalised coordinates and conjugate momenta, in order to justify a particular symplectic structure and corresponding operator algebra. Although it would be possible to define such an extension of GFT, here we simply postulate the basic commutation relations and resulting Fock space, motivated by the origins of GFT in LQG. Alternative constructions leading to a different Fock quantisation are certainly possible.

The definition of the field operators involves a choice of statistics for indistinguishable particles. We choose bosonic statistics, compatible with the picture of spacetime as a condensate. The field operators acting on the Fock space then obey the commutation relations

$$
\left[\hat{\varphi}(g), \hat{\varphi}^{\dagger}\left(g^{\prime}\right)\right]=\mathbb{I}_{R}\left(g, g^{\prime}\right), \quad\left[\hat{\varphi}(g), \hat{\varphi}\left(g^{\prime}\right)\right]=\left[\hat{\varphi}^{\dagger}(g), \hat{\varphi}^{\dagger}\left(g^{\prime}\right)\right]=0
$$

where

$$
\mathbb{I}_{R}\left(g, g^{\prime}\right)=\int_{G} \mathrm{~d} k \prod_{I=1}^{4} \delta\left(g_{I} k\left(g_{I}^{\prime}\right)^{-1}\right)
$$

represents a Dirac delta distribution on the homogeneous space $G^{4} / G$, reflecting the choice of imposing gauge invariance at the level of the field (2.2). Again, for non-compact groups, (2.5) needs to be replaced by a regularised expression, corresponding to a Dirac delta on $G^{4} / G$.

From the commutation relations (2.4), one can now construct a Fock space in the standard way, starting from a Fock vacuum $|0\rangle$. This vacuum is interpreted as a "no-space" state with no quanta, analogous to the Ashtekar-Lewandowski vacuum of LQG. The action of the ladder operators on this state can be naturally interpreted as the creation of a four-valent open spin network vertex; we are interpreting the state

$$
\left|g_{I}\right\rangle=\hat{\varphi}^{\dagger}\left(g_{I}\right)|0\rangle
$$

as a single spin network vertex decorated with the four group elements $g_{1}, \ldots, g_{4}$.

States characterised by nontrivial distributions of the group-theoretic data can be obtained straightforwardly from suitable integrations with wavefunctions,

$$
|f\rangle=\int \mathrm{d} g f\left(g_{I}\right) \hat{\varphi}^{\dagger}\left(g_{I}\right)|0\rangle, \quad f\left(g_{I}\right)=\left\langle g_{I} \mid f\right\rangle=\left\langle 0\left|\hat{\varphi}\left(g_{I}\right)\right| f\right\rangle .
$$

This extends directly to multiparticle states. For these, we can add an additional structure to our interpretation of the states, namely a graph. Consider an $N$-particle state

$$
\left|f_{N}\right\rangle=\frac{1}{\sqrt{N !}} \int(\mathrm{d} g)^{N} f\left(g^{1}, \ldots, g^{N}\right) \prod_{j=1}^{N} \hat{\varphi}^{\dagger}\left(g^{j}\right)|0\rangle .
$$

The wavefunction $f\left(g^{1}, \ldots, g^{N}\right)=\left\langle g_{I}^{1}, \ldots, g_{I}^{N} \mid f_{N}\right\rangle$ will, in general, depend on all $4 N$ group elements, modulo the vertex gauge invariances from (2.2). Notice that, by bosonic symmetrisation, the wavefunction is completely symmetric under the permutation of the tuples of group elements $g_{I}$ associated to each vertex. Such generic $N$-particle states are interpreted graphically as states of $N$ open spin network vertices.

A special class of states, of particular importance in the LQG interpretation, corresponds to the case in which the wavefunction is constructed out of a generic multiparticle wavefunction with 
the application of a projection which reduces the dependence of the wavefunction to products of the type $\left(g_{I}^{i}\right)^{-1} g_{J}^{j}$. This is achieved by $2 N$ convolutions

$$
f\left(\ldots, g_{I}^{i}, \ldots, g_{J}^{j}, \ldots\right)=\int_{G^{2 N}} \mathrm{~d} h_{I J}^{i j} f\left(\ldots, h_{I J}^{i j} g_{I}^{i}, \ldots, h_{I J}^{i j} g_{J}^{j}, \ldots\right) .
$$

Since we are thinking of $g_{I}^{i}$ and $g_{J}^{j}$ as parallel transports of a gauge connection along edges emanating from the vertex to the endpoint, it is natural to interpret such a dependence as the gluing of the edge $I$ incident on the vertex $i$ with the edge $J$ incident on the vertex $j$, with $\left(g_{I}^{i}\right)^{-1} g_{J}^{j}$ being the parallel transport from the vertex $j$ to the vertex $i$ along a connecting edge.

In this way, we can attach to our states a graph label, based on the pattern of convolutions of the arguments, and identify GFT Fock states with a direct relation with the closed spin network wavefunctions in LQG. The precise connection with the (kinematical) LQG states is a delicate issue, and we refer to [105] for a more complete discussion. First, the inner product that we are using here is the Fock space inner product, so that the graph label does not automatically identify orthogonal subspaces of the Fock space: states associated to different graphs, when they have the same number of quanta, are not necessarily orthogonal. Second, wavefunctions are completely symmetrised with respect to the permutations of vertices, while in the case of LQG the wavefunctions do not necessarily have to have special symmetrisation properties. Finally, GFT Fock states are not organised in terms of embedded graphs. The graphs we can associate to them are purely combinatorial objects, and make no reference to any background manifold structure, except to the group manifolds from which the data decorating the graphs are picked. Thus, they are closer to the setting of algebraic quantum gravity [73, 74], an approach for defining canonical LQG without using embeddings or a differentiable manifold structure.

Keeping in mind these subtleties, the Fock space language allows us to import many of the concepts of LQG into the GFT setting [105]. In particular, one can immediately translate the operators that are normally defined for spin networks into the second quantised language that we have introduced. In order to do so, we now consider the case $G=\mathrm{SU}(2)$, the gauge group of the Ashtekar-Barbero formulation of classical general relativity $[5,19]$ that is the basis of LQG.

The translation requires identifying the product of ladder operators that corresponds to the first quantised operators of interest. Hermitian operators are of particular significance, as they will store information about observables. In order to be Hermitian, these operators have to be built with (linear combinations of) strings of ladder operators possessing an equal number of creation and annihilation operators. According to the number of creation operators, operators will be distinguished as one-body operators, two-body operators, and so on. The definition of these operators is necessary to extract geometric information from states containing arbitrary superpositions of states associated to different graphs. The translation of intrinsically manybody operators (as, for instance, the holonomy computed on a loop incident on several vertices) is not immediate, as the nature of the GFT quanta as identical particles makes the identification of portions of the graphs a tricky issue, in absence of further degrees of freedom that can be used to distinguish between different kinds of vertices. Such degrees of freedom could, for example, correspond to matter fields, or to an additional "colour" label of multiple GFT fields (see Section 3.5 for some more discussion on colouring in GFT). For our purposes, one-body operators are enough to extract the relevant geometric information.

Generic one-body Hermitian operators have the form

$$
\widehat{X}=\int \mathrm{d} g \mathrm{~d} g^{\prime} X\left(g, g^{\prime}\right) \hat{\varphi}^{\dagger}(g) \hat{\varphi}\left(g^{\prime}\right), \quad X\left(g, g^{\prime}\right)=\overline{X\left(g^{\prime}, g\right)} .
$$

The simplest one-body operator is the number operator $\widehat{N}$, with $X=1$,

$$
\widehat{N}=\int \mathrm{d} g \hat{\varphi}^{\dagger}(g) \hat{\varphi}(g) .
$$


This operator extracts a specific and unambiguous graph property, the number of vertices, or the average number of vertices in the case of a superposition of graphs.

Other common one-body operators that are relevant in the GFT context are the vertex volume operator $\widehat{V}$ and the plaquette area $\widehat{A}_{I}$. They can be obtained by simply setting as $X\left(g, g^{\prime}\right)$ the LQG matrix elements of the desired operators, e.g.,

$$
\widehat{V}=\int \mathrm{d} g \mathrm{~d} g^{\prime} V^{\mathrm{LQG}}\left(g, g^{\prime}\right) \hat{\varphi}^{\dagger}(g) \hat{\varphi}\left(g^{\prime}\right),
$$

where $V^{\mathrm{LQG}}\left(g, g^{\prime}\right) \equiv\left\langle g_{I}\left|V^{\mathrm{LQG}}\right| g_{I}^{\prime}\right\rangle$ is the matrix element of the volume operator between singlevertex spin networks in LQG, and (replacing the matrix elements by a differential operator)

$$
\widehat{A}_{I}=\int \mathrm{d} g \hat{\varphi}^{\dagger}(g)\left(\sum_{m, n=1}^{3} \delta_{m n} \mathrm{E}_{I}^{m} \mathrm{E}_{I}^{n}\right)^{1 / 2} \triangleright \hat{\varphi}(g) .
$$

Here $\mathrm{E}_{I}^{n}$ are the LQG flux operators, proportional to the Lie derivatives on $\mathrm{SU}(2)$,

$$
\mathrm{E}_{I}^{n} f\left(g_{J}\right)=\lim _{\epsilon \rightarrow 0} \mathrm{i} \kappa \frac{d}{d \epsilon} f\left(e^{-\mathrm{i} \sigma^{n} \epsilon / 2} g_{I}\right),
$$

where $\sigma^{n}$ is a Pauli matrix and the operator only acts on the $I$-th argument of the field, and $\kappa$ is a parameter with dimensions of area, the "Planck area" of the theory, which in canonical LQG is usually set to $\kappa=8 \pi \gamma \hbar G_{\mathrm{N}}$ where $\gamma$ is the Barbero-Immirzi parameter and $G_{\mathrm{N}}$ is the low-energy Newton's constant ${ }^{1}$. Noting that contracting the Lie derivatives with the Killing form leads to the Casimir operator on $\mathrm{SU}(2),(2.7)$ can be simplified to

$$
\widehat{A}_{I}=\kappa \int \mathrm{d} g \hat{\varphi}^{\dagger}(g) \sqrt{-\Delta_{g_{I}}} \hat{\varphi}(g),
$$

where $\Delta_{g_{I}}$ is the Laplace-Beltrami operator on SU(2) acting on the $I$-th argument of $\hat{\varphi}$. This shows explicitly how the (kinematical) area spectrum in LQG is related to the discrete spectrum of the Laplacian on $\mathrm{SU}(2)$, multiplied by the parameter $\kappa$.

A basic property of one-body operators is that they are extensive: as it is immediate to realise using Wick's theorem, they generate a contribution from each of the quanta of the state on which they act. This aspect will play a role in our discussion of the condensate states and their geometrical content; by only using one-body operators in extracting geometric data from the states, we focus on quantities that are local at the level of each vertex or dual tetrahedron.

\subsection{Noncommutative dual formulation of GFT}

Group field theories can be formulated in different equivalent representations, using suitably defined Fourier transforms. The simplest one is the spin representation (using the terminology for $\mathrm{SU}(2)$ ), which is based on a Peter-Weyl decomposition. A slightly more sophisticated representation of the theory is the one that makes uses of noncommutative fluxes (see, e.g., $[15,77])$. The basic idea is to use a noncommutative Fourier transform that unitarily maps square-summable functions on the group to square-summable functions on the corresponding Lie algebra $\mathfrak{g}$,

$$
\mathcal{F}(f)(B) \equiv \int_{G} \mathrm{~d} g \mathrm{e}_{g}(B) f(g),
$$

where $\mathrm{e}_{g}(B)$ are plane waves associated to the particular quantisation map chosen [77].

\footnotetext{
${ }^{1}$ Introducing $G_{\mathrm{N}}$ in this way, at the kinematical level in the microscopic theory, raises the question of whether $G_{\mathrm{N}}$ can undergo renormalisation in LQG. The results in the later sections of this review will show how, in GFT condensate cosmology, the strength of the gravitational coupling to matter emerges from the coupling constants of the GFT action, so that $G_{\mathrm{N}}$ has a rather different origin and is subject to renormalisation.
} 
This allows a definition of Fourier transformed field operators acting on the same Fock space,

$$
\hat{\tilde{\varphi}}\left(B_{I}\right)=\int \mathrm{d} g \prod_{I=1}^{4} \mathrm{e}_{g_{I}}\left(B_{I}\right) \hat{\varphi}\left(g_{I}\right),
$$

where, corresponding to the four group elements $g_{I}, B_{I}=\left(B_{1}, \ldots, B_{4}\right)$ with $B_{I} \in \mathfrak{g}$.

The peculiarity of the Lie algebra representation is that, instead of the ordinary product, a noncommutative star-product has to be used in order to encode the noncommutative group multiplication. This product is defined in terms of its action on plane waves,

$$
\mathrm{e}_{g}(B) \star_{B} \mathrm{e}_{h}(B)=\mathrm{e}_{g h}(B) .
$$

While the Lie algebra representation presents a number of technical difficulties due to the nontrivial aspects of the star-product, it offers a dual, and perhaps clearer geometric interpretation of the GFT quanta. Let us specialise again the discussion to $G=\mathrm{SU}(2)$, where the Lie algebra variables $B_{I}$ can be naturally identified with the densitised triads integrated over the plaquettes dual to the edges attached to a GFT vertex, see $(3.7)^{2}$.

This interpretation is corroborated by the following observation. Using the (noncommutative) Dirac delta distribution in the Lie algebra representation, given by

$$
\delta_{\star}(B)=\int_{G} \mathrm{~d} g \mathrm{e}_{g}(B),
$$

we can easily see that the right gauge invariance of the group representation translates into a closure condition of the fluxes, as

$$
\begin{aligned}
\hat{\tilde{\varphi}}\left(B_{I}\right) & =\int \mathrm{d} g \int_{G} \mathrm{~d} h \prod_{I=1}^{4} \mathrm{e}_{g_{I}}\left(B_{I}\right) \hat{\varphi}\left(g_{I} h^{-1}\right)=\int \mathrm{d} g \int_{G} \mathrm{~d} h \prod_{I=1}^{4} \mathrm{e}_{g_{I}}\left(B_{I}\right) \hat{\varphi}\left(g_{I}\right) \\
& =\hat{\tilde{\varphi}}\left(B_{I}\right) \star_{B_{I}} \delta_{\star}\left(\sum_{I=1}^{4} B_{I}\right) .
\end{aligned}
$$

This closure condition $\sum_{I} B_{I}=0$ suggests that the state

$$
\left|B_{I}\right\rangle=\hat{\tilde{\varphi}}^{\dagger}\left(B_{I}\right)|0\rangle
$$

can be interpreted as a closed tetrahedron, with $B_{I}$ being the bivector of the face $I$.

The noncommutative counterpart of the convolution of group elements (2.6) that leads to edges connecting different vertices is the insertion of a noncommutative Dirac delta, $\delta_{\star}\left(B_{I}^{i}+B_{J}^{j}\right)$, as can be seen by Fourier transforming (2.6). The insertion of a star-Delta function now can be interpreted as imposing the condition that the tetrahedron $i$ and the tetrahedron $j$ are glued along the faces $I$ and $J$, respectively. The fact that they are identified requires that the corresponding geometric data are properly matched, thus the condition $B_{I}^{i}=-B_{J}^{j}$.

In this way, states built with the noncommutative dual representation are naturally associated to three-dimensional simplicial complexes decorated with data that are directly related to the intrinsic Riemannian geometry. Therefore, they can be seen as triangulations of the spatial slices used in the computation of quantum gravity amplitudes. It should be kept in mind, however,

\footnotetext{
${ }^{2}$ The construction of a noncommutative Fourier transform, used for the specific case of SU(2) (and hence Spin(4)), can be extended, at least formally, to the case of weakly exponential Lie groups [77], including SL(2, C). What is so far missing is the explicit construction of the plane waves and the detailed analysis of their properties and ambiguities (related to the choice of a quantisation map) in their definition [77]. Hence, so far there is no explicit construction of Lorentzian models based on flux variables. However, there is also no obvious reason why such a construction should not be possible.
} 
that what we are gluing together are quantum tetrahedra. This will be evident whenever we will try to compute the action of geometric operators on these states. Despite the subtleties related to the noncommutative nature of the Lie algebra variables, the flux representation allows a more transparent construction of states having specific continuum metric properties, for example corresponding to a particular spatially homogeneous metric as relevant in cosmology.

\subsection{Equations of motion in second quantisation}

The tools that we have introduced can be used to design interesting states, which can be interpreted in terms of spatial three-dimensional geometries. The last ingredient that we need is the one that will generate the sum over four-dimensional discrete geometries. This is achieved again in terms of the perturbative expansion of a suitable dynamical field equation. As a simple option, we can use the field equation from a classical action used in the partition function,

$$
\frac{\delta S\left[\hat{\varphi}, \hat{\varphi}^{\dagger}\right]}{\delta \hat{\varphi}(g)}|\Phi\rangle=0
$$

but other choices are possible. What is important is that the field equation selecting the physical state $|\Phi\rangle$ can be split into a free part, which admits for instance the Fock vacuum as a solution, and an interaction term with the correct combinatorics.

The perturbative expansion of the partition function is now translated into the expansion of $|\Phi\rangle$ in powers of a coupling constant controlling the interaction term. Following the procedures of standard time-independent perturbation theory in quantum mechanics, one then recovers a diagrammatic expansion of the various correlation functions in the field theory. To be more precise, in the previous sections we have seen how convolutions of field operators can be interpreted in terms of discrete geometric quanta, glued together to form specific geometrical structures. Consider then two sets of strings of creation operators, each associated to a graph, $\Gamma_{1}, \Gamma_{2}$, with data encoded in wavefunctions $f_{1}, f_{2}$. Let us denote these operators as $\widehat{\Psi}\left(\Gamma_{i}, f_{i}\right)$,

$$
\widehat{\Psi}\left(\Gamma_{i}, f_{i}\right)=\int(\mathrm{d} g)^{\# V\left(\Gamma_{i}\right)} f_{i}\left(g^{i}, \Gamma_{i}\right) \prod_{j=1}^{\# V\left(\Gamma_{i}\right)} \hat{\varphi}^{\dagger}\left(g^{j}\right) .
$$

It is easy to see that a correlation function such as

$$
G\left(\Gamma_{1}, f_{1} ; \Gamma_{2}, f_{2}\right)=\frac{\left\langle\Phi\left|\widehat{\Psi}^{\dagger}\left(\Gamma_{1}, f_{1}\right) \widehat{\Psi}\left(\Gamma_{2}, f_{2}\right)\right| \Phi\right\rangle}{\langle\Phi \mid \Phi\rangle},
$$

once the physical vacuum is treated perturbatively $|\Phi\rangle=|0\rangle+\sum_{i=1}^{\infty} \lambda^{i}\left|\Phi^{(i)}\right\rangle$, can be rewritten in terms of a diagrammatic expansion, that can be interpreted in terms of four-dimensional discrete geometries having as boundaries the three-dimensional geometries dual to the graphs $\Gamma_{1}, \Gamma_{2}$, with the proper inclusion of bulk and boundary geometric data, as depicted schematically in Fig. 3 for the case of a lower-dimensional theory.

The choice of the ingredients appearing in (2.9) (ladder operators and kernels) will then specify which graphs are included in the perturbative expansions of the correlation functions such as (2.10), together with the corresponding amplitudes. Gravitational models can be designed so that (2.9) leads to graphs and amplitudes that match the ones of the spin foam expansion.

The correlation functions in (2.10) should not directly be interpreted in terms of transition amplitudes, as there is no notion of time evolution in this timeless framework. They should be viewed instead as the analogue of the matrix elements of the rigging map of LQG, enforcing the constraints that map kinematical states into physical states. 

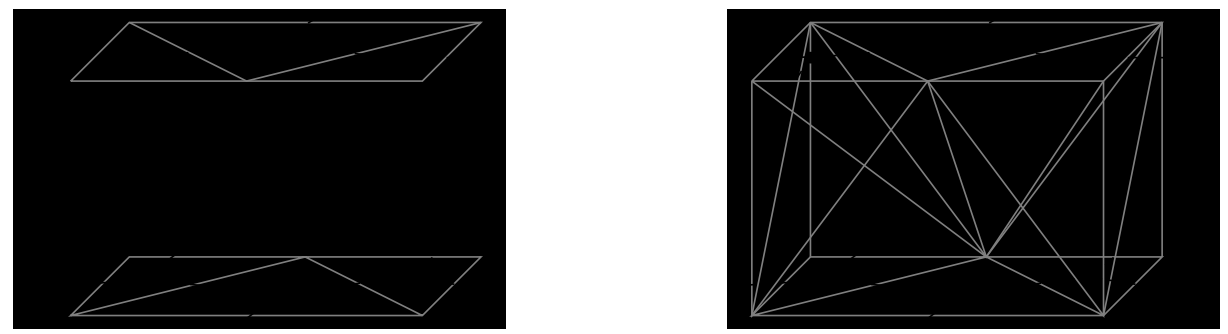

Figure 3. Given two boundary states, the perturbative expansion of the correlation function (2.10) will generate all the simplicial complexes compatible with them, and the corresponding amplitude.

As in the functional formulation, a proper definition of $|\Phi\rangle$ must pass through the examination of the perturbative series and its renormalisability, with all the related problems. The true advantage of an operatorial formulation is the possibility of developing a whole new approximation scheme for $|\Phi\rangle$ that is not based on a perturbation expansion, and this is the reason why we prefer this formulation of the theory.

If we view the theory defined in (2.9) as a peculiar many-body problem of the kind encountered in condensed matter physics, we can try to abandon the perturbative expansion of the physical state and introduce a completely different approximation, based on a nonperturbative trial state $\left|\Phi_{\text {trial }}\right\rangle$ that will include our guesses about the nature of the physical state of the theory, which will be presumably very different from the Fock vacuum that is used for the perturbative expansion. This is the point of view adopted when we are dealing with the GFT condensates, as it will be explained. It will be also manifest how this approach will make the investigation of the regime in which a large number of quanta are involved much more productive.

\subsection{Relation of GFT to tensor models and spin foam models}

We have already mentioned that GFT is closely related to other approaches to the statistical mechanics of random geometries.

The case of matrix models for random surfaces [51] is perhaps the case that is understood with the greatest level of detail. The partition function, for a finite size matrix model, is in fact an ordinary integral, and there are many different techniques that can be used to determine its behaviour. The critical properties of the model, describing the continuum limit of these models for discrete random surfaces, can be extracted from a properly defined scaling limit, in which the coupling constants are tuned to critical values while the matrix size is sent to infinity.

Tensor models (see [80, 122] for reviews) are higher-dimensional generalisations of matrix models. They involve tensorial objects with higher rank, whose combinatorics is rich enough to permit the construction of Feynman diagrams that can be put in correspondence with simplicial complexes. As in the case of matrix models, tensor models are dealing only with the combinatorial structure of the Feynman diagrams. In recent years, a variety of results have been gathered about the combinatorial aspects of the partition function of tensor models, leading to a first understanding of their critical behaviour [40, 79].

There is a strong relationship between tensor models and GFT. In a certain sense, one could view GFTs as tensor models with indices running on a continuous set, a Lie group, instead of a discrete set. Conversely, a tensor model might be pictured as a GFT based on a discrete group as $\mathbb{Z}_{N}$, for instance. More generally, for compact groups, one can rewrite, via the PeterWeyl decomposition, a GFT as a generalised tensor model involving various tensorial degrees of freedom, labelled by the irreducible unitary representations of the given group (with a cutoff to obtain a finite index range). This reduces to a conventional tensor model for $G=\mathrm{U}(1)$. 
GFT makes use of the same principles of the combinatorics of tensor models. In addition, however, the additional group-theoretic data give rise to a much richer variety of models, at fixed combinatorics, corresponding to the possible ways to use this data to build the amplitudes. Most significantly, the additional data of GFT allow us to construct models which can be rewritten as simplicial path integrals for first-order gravitational theories [16, 17], in particular Plebanskitype reformulations of general relativity that are adapted to the Ashtekar variables for LQG.

The analysis of the GFT partition function then has to go beyond the combinatorial aspects, and presents additional difficulties with respect to the partition function for matrix and tensor models. Indeed, the GFT formalism provides additional data in the amplitudes of the graphs, which are no longer characterised by the combinatorial structure alone. Therefore, the considerations leading to the identification of the classes of graphs responsible for the dominant contributions to the partition functions need to be revised. The hope is that the number of results obtained in the case of tensor models, particularly on the problem of renormalisability, phase transitions and the continuum limit, might be ultimately adapted and extended to GFT. The renormalisation of GFT is an ongoing research programme (see Section 1.2).

While GFT actions can obviously be of very general shapes, the special class built on generalisations of the Ooguri model plays an important role for the construction of candidates for quantum gravity models. The reason for this is the precise relationship between GFT and spin foam models, a proposal for the definition of a discretised sum-over-histories version of quantum gravity. In turn, spin foams are closely related to loop quantum gravity, as spin foam amplitudes are related to histories of spin networks. In four spacetime dimensions, the question of whether spin foams and canonical LQG can be seen as defining equivalent theories is a subtle issue and a focus of current research [3]. The GFT formalism gives a different perspective on this issue: the canonical and covariant descriptions of GFT simply correspond to the standard dichotomy of defining a quantum field theory through a Fock space/operator formalism or through the path integral. The two viewpoints on GFT can then be respectively be related to canonical LQG and spin foam models, without the need to directly map between canonical LQG and spin foams.

It is known that classical Einstein gravity without a cosmological constant can be formulated in a variety of ways using different sets of degrees of freedom and different variational principles (see, e.g., [116]). The Plebanski formulation starts from a topological BF theory and supplements it with a term imposing a certain set of constraints. These constraints make the theory equivalent to a Palatini (i.e., a first order) formulation of vacuum general relativity. Indeed, the role of these constraints is to break the gauge symmetries of the topological theory, which would not possess the local degrees of freedom required by general relativity ${ }^{3}$.

The spin foam approach to quantum gravity takes advantage of these results. The basic idea is to start from the quantisation of BF theories, suitably discretised on a cellular complex, and then to impose the necessary constraints that would turn a topological theory into a gravitational theory [12]. In the topological theory, the data associated to the complexes (typically expressed in terms of representation labels) are allowed to take values in the whole range allowed by the gauge group involved, $\operatorname{Spin}(4)$ or $\mathrm{SL}(2, \mathbb{C})$. The imposition of the simplicity constraints restricts the allowed data that can be attached to a spin foam. In particular, when looking at the Lie algebra data, they are equivalent to the requirement that these data can be rewritten in terms of discretised tetrad fields. The various proposals in the literature try to achieve this goal using a variety of related but different ideas and techniques [117].

There is a precise correspondence between spin foam models and GFT. For any given spin foam model, one can find a corresponding GFT action in a precise way [121]: given the amplitude assigned to any given cellular complex by the spin foam model, there is a GFT action whose Feynman graphs, dual to cellular complexes, have matching amplitudes. Seen from the opposite

\footnotetext{
${ }^{3}$ It is worth mentioning that the three-dimensional case does not require the introduction of constraints, as three-dimensional general relativity without a cosmological constant can be written as a topological BF theory.
} 
direction, a GFT action defines a spin foam model, once the partition function is rewritten in terms of the Feynman expansion,

$$
Z=\int \mathcal{D} \varphi \mathcal{D} \bar{\varphi} e^{-S[\varphi, \bar{\varphi}]}=\sum_{\sigma} \frac{\prod_{i}\left(\lambda_{i}\right)^{N_{i}(\sigma)}}{\operatorname{Aut}(\sigma)} \mathcal{A}_{\sigma} .
$$

Here we are labelling the Feynman graphs with the corresponding dual cellular complexes $\sigma$, in order to make the correspondence with spin foam approach more transparent. We are also including the possibility that several different interactions terms are introduced, each with different kernels and different combinatorial structure, and each controlled by a different coupling constant $\lambda_{i} . N_{i}(\sigma)$ will be then the total number of vertices of type $i$ appearing in the Feynman diagram. Aut $(\sigma)$ is the order of the automorphisms of $\sigma$. This is the combinatorial part of the amplitude. It can be shown by direct calculations that the amplitude $\mathcal{A}_{\sigma}$, the Feynman amplitude that the GFT model assigns to $\sigma$, can be represented as a spin foam model.

An important ingredient that is explicitly added by the GFT approach is a definite prescription for generating weights for amplitudes for different cellular complexes in the sum over $\sigma$. These depend on the coupling constants of the GFT action, as (2.11) shows explicitly.

We now present a concrete implementation of the ideas sketched so far, restricting the analysis to the case of models and data relevant for four-dimensional gravity. The starting point is the Ooguri model for $\operatorname{Spin}(4)$ or $\mathrm{SL}(2, \mathbb{C})$, which will be modified to include the restriction on the geometric data only. There are several similar but inequivalent constructions, as the imposition of simplicity constraints is not uniquely specified by the classical theory. Indeed, the translation of the classical, continuum constraints into the discrete setting faces various discretisation and ordering ambiguities related to the non-Abelian nature of the variables. Furthermore, the simplicity constraints are characterised by a free parameter, the Barbero-Immirzi parameter.

The idea of GFT and spin foam models is to work with graphs decorated with the data of $\mathrm{SL}(2, \mathbb{C})$ or Spin(4) BF theories, and then to restrict the sums to only those data that correspond to the geometric degrees of freedom. For a finite value of the Barbero-Immirzi parameter, one way to proceed is as follows. It is convenient to work with a field theory based on $\mathrm{SU}(2)$, so that we can work directly with the geometric data and use the tools that have been built to deal with LQG spin networks. Following [60], we can define a map $S$ from the space of functions of $\mathrm{SU}(2)$ into the space of functions of $\operatorname{Spin}(4)$ (or $\mathrm{SL}(2, \mathbb{C}))^{4}$. Denoting with $G_{I}$ the elements of the four-dimensional gauge group, the $\mathrm{SU}(2)$ field is mapped into

$$
(S \varphi)\left(G_{I}\right)=\int \mathrm{d} g S\left(G_{I}, g_{J}\right) \varphi\left(g_{J}\right) .
$$

This map $S$ takes care of restricting the Spin(4) data for the Feynman diagrams to geometric data only, and it is the part of the construction where the implementation of the simplicity constraints, encoded in the so-called fusion coefficients [117], is located. The familiar expressions for the various spin foam models can be recovered by expanding these kernels in the appropriate basis functions (Wigner representation matrices, coherent states, noncommutative plane waves ${ }^{5}$ ), but the explicit form of this kernel is not very illuminating.

The interaction term for the Ooguri model for Spin(4), built with (2.3), can be used to define the interaction term for the $\mathrm{SU}(2)$ theory,

$$
\mathcal{V}_{\mathrm{SF}}[\varphi, \bar{\varphi}]=\mathcal{V}_{\text {Ooguri }}[S \varphi, \overline{S \varphi}]
$$

\footnotetext{
${ }^{4}$ Although, for the cases of interest, in the $\operatorname{SL}(2, \mathbb{C})$ case they would be distributions.

${ }^{5}$ As discussed in [16, 17], it is possible to build GFT actions exploiting the relationship between their flux representation, based on the noncommutative Fourier transform discussed in Section 2.3, and simplicial path integrals that make direct use of flux variables.
} 
The presence of the maps $S$ will then guarantee that, in the Feynman diagrams, only the Spin(4) data solving the simplicity constraints will be summed over. The Lorentzian case proceeds along similar lines, although some additional care has to be used as the various expressions might require regularisation, before they can be manipulated.

While this is not the only possible way to proceed, it is the simplest one that allows us to work directly with geometric data and with the familiar $\mathrm{SU}(2)$ spin networks. This choice will also make it easier to keep track of the differences between different prescriptions for the simplicity constraints. For the Barrett-Crane model, which corresponds to infinite Immirzi parameter, the construction is slightly different as $\mathrm{SU}(2)$ has to be replaced with the homogeneous space $\mathrm{SL}(2, \mathbb{C}) / \mathrm{SU}(2)$ (or $\mathrm{Spin}(4) / \mathrm{SU}(2) \simeq \mathrm{SO}(4) / \mathrm{SO}(3) \simeq \mathrm{SU}(2)$ in the Riemannian case) [50].

\section{GFT condensates: kinematics}

After this discussion of the general formalism of GFT in terms of kinematics, dynamics, and the relation to LQG and spin foams, in this section we review the detailed construction and interpretation of condensate states in GFT. There are close similarities to how one proceeds in the case of a non-relativistic quantum field theory describing Bose-Einstein condensates [118], but also important differences due to the fact that we are dealing with a field theory of quantum geometry as a (background-independent) proposal for quantum gravity. We largely follow the constructions and discussion given in the original papers [71, 72], on which most of the work so far has been based. In Section 3.5 we will show how these constructions can be generalised and extended to states that might be closer to those of physical interest in quantum gravity, and that have the advantage of also describing situations of spherical symmetry.

\subsection{Definitions and simplest condensates}

A condensate phase of a quantum field theory can be characterised by an order parameter, associated to a non-zero expectation value of a field operator in this phase which would be zero in the standard Fock vacuum. In the simplest case, this order parameter can be associated with the "condensate wavefunction", i.e., a complex-valued function on the configuration space of the basic quantum field that fully determines the quantum state.

More concretely, we can focus on a particular subset of the space of general $N$-particle states

$$
|\Psi\rangle:=\int(\mathrm{d} g)^{N} \Psi\left(g_{I}^{1}, \ldots, g_{I}^{N}\right) \bigotimes_{i=1}^{N}\left|g_{I}^{i}\right\rangle
$$

in which the wavefunction $\Psi$ decomposes into a product of functions depending on fewer arguments. Physically, this means neglecting correlations between different particles either altogether or beyond a certain order (e.g., beyond 2-particle or 3-particle correlations). The two simplest cases are of particular interest: in the first, $\Psi$ decomposes into one-particle wavefunctions,

$$
\Psi\left(g_{I}^{1}, \ldots, g_{I}^{N}\right)=\prod_{i=1}^{N} \psi\left(g_{I}^{i}\right)
$$

whereas in the second it involves two-particle correlations (this is known as the Bogoliubov approximation in the context of Bose-Einstein condensates [118]),

$$
\Psi\left(g_{I}^{1}, \ldots, g_{I}^{N}\right)=\prod_{i=1}^{N / 2} \psi\left(g_{I}^{2 i-1}, g_{I}^{2 i}\right)+\text { permutations }
$$


where $N$ is even and the permutations are needed to ensure that the wavefunction $\Psi$ is symmetric under the exchange of any two vertex indices, compatible with the bosonic statistics for the GFT. This symmetry will be automatically implemented once bosonic field operators are used.

States of the type (3.1) or (3.2) could be used as simple approximations to a condensate, and capture much of the relevant physics of condensates. However, they do not satisfy all of the desired properties; in particular, given that they describe states of fixed particle number, the expectation value of the field operators $\hat{\varphi}$ and $\hat{\varphi}^{\dagger}$ vanish. We hence need to use states that are superpositions of states of the type (3.1) or (3.2) for different $N$, with appropriate weights in the sum, and are led to the definition of a single-particle condensate state

$$
|\sigma\rangle:=\mathcal{N}(\sigma) \exp \left(\int \mathrm{d} g \sigma\left(g_{I}\right) \hat{\varphi}^{\dagger}\left(g_{I}\right)\right)|0\rangle,
$$

where $\mathcal{N}(\sigma)=\exp \left(-\frac{1}{2} \int \mathrm{d} g\left|\sigma\left(g_{I}\right)\right|^{2}\right)$ is a normalisation factor, and a dipole condensate state

$$
|\xi\rangle:=\mathcal{N}(\xi) \exp \left(\int \mathrm{d} g \mathrm{~d} h \xi\left(g_{I}^{-1} h_{I}\right) \hat{\varphi}^{\dagger}\left(g_{I}\right) \hat{\varphi}^{\dagger}\left(h_{I}\right)\right)|0\rangle
$$

with $\mathcal{N}(\xi)$ again a normalisation factor (given explicitly in [72]). Note the definition of $\xi$ in (3.4), instead of an in principle possible more general wavefunction $\psi\left(g_{I}, h_{I}\right)$. Because $\hat{\varphi}^{\dagger}\left(g_{I}\right)=$ $\hat{\varphi}^{\dagger}\left(g_{I} h\right)$, the functions $\sigma$ and $\xi$ satisfy $\sigma\left(g_{I}\right)=\sigma\left(g_{I} h\right)$ and $\xi\left(g_{I}\right)=\xi\left(k g_{I} h\right)$ for all $h, k \in G$. For (3.3) to also represent a state invariant under a "gauge transformation" acting simultaneously on the open ends of the spin network represented by an open vertex, we also impose $\sigma\left(g_{I}\right)=$ $\sigma\left(k g_{I}\right)$ for all $k \in G$. (3.4) is built out of elementary "dipoles" that correspond to gauge-invariant states in LQG, and are characterised by a single wavefunction $\xi\left(g_{I}\right)$ on $G \backslash G^{4} / G$, as would be the possible states on a "dipole" in usual LQG [125].

The states (3.3) and (3.4) satisfy the property of wavefunction homogeneity which requires the state to be determined by a single-particle wavefunction $(\sigma$ or $\xi)$. They are superpositions of states of the type (3.1) and (3.2) for different particle numbers, and hence rather different from the states usually considered in LQG which are typically built on a fixed graph.

An important property of the state (3.3) is that it is an eigenstate of the GFT field operator,

$$
\hat{\varphi}\left(g_{I}\right)|\sigma\rangle=\sigma\left(g_{I}\right)|\sigma\rangle \text {. }
$$

From this it immediately follows that $\left\langle\hat{\varphi}\left(g_{I}\right)\right\rangle=\sigma\left(g_{I}\right)$, so that $\sigma\left(g_{I}\right)$ represents the order parameter, and that more generally, any normal-ordered $n$-point function involving $\hat{\varphi}\left(g_{I}\right)$ and $\hat{\varphi}^{\dagger}\left(g_{I}\right)$ can be evaluated by replacing $\hat{\varphi}$ by $\sigma$ and $\hat{\varphi}^{\dagger}$ and $\bar{\sigma}$. In this very simplest approximation, there are no correlations between the GFT quanta, and in the mean-field approximation when fluctuations can be ignored, $\sigma\left(g_{I}\right)$ directly represents a classical GFT field configuration. This property will be important in Section 3.3 when the geometric interpretation of (3.3) will be discussed.

As is apparent from the appearance of an explicit normalisation factor in (3.3) and (3.4), these states are normalisable for appropriate choices of the functions $\sigma$ and $\xi$, and hence elements of the GFT Fock space defined in Section 2. For (3.3), normalisability of the state is equivalent to a finite expectation value of the number operator,

$$
N:=\langle\sigma|\widehat{N}| \sigma\rangle=\left\langle\sigma\left|\int \mathrm{d} g \hat{\varphi}^{\dagger}\left(g_{I}\right) \hat{\varphi}\left(g_{I}\right)\right| \sigma\right\rangle=\int \mathrm{d} g\left|\sigma\left(g_{I}\right)\right|^{2}<\infty .
$$

The thermodynamic limit $N \rightarrow \infty$ is described by states that are no longer in the original GFT Fock space: this is standard in quantum field theory, where in the limit corresponding to a phase transition one needs to change representation to a different, unitarily inequivalent Hilbert space. Here $N \rightarrow \infty$ corresponds to a continuum limit in which such a transition is reached. 
In practice, the difference between fixed particle number as in (3.1) and fluctuating particle number as in (3.3) becomes unimportant for large $N$ : an elementary calculation shows that

$$
(\Delta N)_{|\sigma\rangle}:=\sqrt{\left\langle\sigma\left|\widehat{N}^{2}\right| \sigma\right\rangle-(\langle\sigma|\widehat{N}| \sigma\rangle)^{2}}=\sqrt{\langle\sigma|\widehat{N}| \sigma\rangle}=\sqrt{N}
$$

and hence the relative uncertainty $(\Delta N) / N \sim 1 / \sqrt{N}$ vanishes in the thermodynamic limit; this is the well-known equivalence of the canonical and grandcanonical ensembles in this limit.

The dipole condensate state (3.4) is no longer a coherent state for the GFT field operator, but closer to squeezed states that appear in quantum optics [96]. While a refinement of (3.3) that incorporates the usual notion of gauge invariance in LQG and contains two-particle correlations, is already harder to do computations with; no simple closed expression for general $n$-point functions or quantities such as the average particle number is known. Approximate expressions for correlation functions are discussed in [72], and can be used for estimates of the error that one makes in using them.

The definition of the states (3.3) and (3.4) is mainly motivated by their nice properties from the perspective of quantum field theory and by the analogy to the well-studied case of Bose-Einstein condensates. They assume a truncation to one- and two-particle correlations respectively, which can be seen as corresponding to the situation of cosmological relevance, where we focus on geometries that are spatially homogeneous or nearly homogeneous, so that spatial gradients can be ignored in a first approximation. However, the truncation also means that these states cannot be interpreted in the sense of an extended graph in LQG: they would correspond to "graphs" consisting of a large number of disconnected components formed by a single vertex or dipole, respectively. Any topological information normally also contained in the graph must come from elsewhere. One way to remedy this situation is to define generalised condensates that are associated to a graph of fixed topology, as we will outline in Section 3.5. Another is to make an assumption for the topology of space and require self-consistency with the dynamics: e.g., if the effective dynamics of isotropic and homogeneous geometries is given by the Friedmann equation with positive spatial curvature, one infers that space should have the topology of a 3-sphere. This viewpoint, advocated in [72] and in some sense closer to the formalism of canonical LQG [124, 131], will be outlined in Section 3.3.

\section{2 "Condensate wavefunction" vs. "wavefunction of the universe"}

The mean field $\sigma\left(g_{I}\right)$ appearing in the definition (3.3) is often referred to as the "condensate wavefunction". Indeed, it is a wavefunction in the sense that $|\sigma\rangle$ is a superposition of states with all possible particle numbers, each of which is defined by a product wavefunction of the form (3.1) where $\psi$ is replaced by $\sigma: \sigma$ is the wavefunction of the "ground state" that the GFT quanta are supposed to be condensed into (again, in this mean-field approximation where fluctuations over this condensed phase are ignored).

In most other respects, it can be quite misleading to think of $\sigma$ as a usual quantum-mechanical wavefunction. First of all, the overall normalisation of $\sigma$ is not arbitrary as in quantum mechanics, but corresponds to the average particle number (see (3.5)). Indeed it is often useful to adopt a parametrisation $\sigma\left(g_{I}\right)=\sqrt{N} \sigma_{0}\left(g_{I}\right)$ where $\sigma_{0}$ is normalised to one [118].

More importantly, there is no superposition principle and no direct probability interpretation associated with $\sigma\left(g_{I}\right)$, as the dynamics of $\sigma$ is typically governed by nonlinear (and nonlocal) equations. At first sight, this may appear confusing: are we proposing a radical nonlinear, nonlocal modification of quantum mechanics for GFT? It should be clear, and will become clearer in Section 4 below, that this is not the case, and all dynamical equations are linear equations on the GFT Fock space. However, the correspondence between a condensate state and its "wavefunction" is clearly nonlinear,

$$
|\sigma\rangle+\left|\sigma^{\prime}\right\rangle \neq\left|\sigma+\sigma^{\prime}\right\rangle
$$


and linear equations on the Hilbert space become nonlinear equations for $\sigma$. Whatever the precise form of the effective dynamics for $\sigma$, it will not make sense to directly interpret them as a Schrödinger-type or Wheeler-DeWitt-type equation for a "wavefunction of the universe" as in standard quantum cosmology [134], and common tools in quantum cosmology such as the WKB approximation will have to be reinterpreted according to the rôle of $\sigma$ as a mean-field configuration of the quantum field, rather than a single-particle ("single-universe") wavefunction.

To make this more precise, let us compare with the situation in condensed matter physics. Here the "condensate wavefunction" describing the simplest approximation to the Bose-Einstein condensate can be written as [118]

$$
\Psi(\vec{x})=\sqrt{\rho(\vec{x})} \exp (-\mathrm{i} \theta(\vec{x}))
$$

where $\rho(\vec{x})$ defines the density and $\theta(\vec{x})$ the velocity potential (for irrotational fluids, the velocity is $\vec{v} \propto \vec{\nabla} \theta)$ of the superfluid formed by the condensate. $\Psi$ clearly has no probability interpretation; it already describes a classical limit, the mean-field approximation, in which it corresponds to a classical field configuration (given by two real functions $\rho, \theta$ or one complex function $\Psi)$.

A WKB approximation applied to (3.6) would correspond to a regime in which the fluid density varies slowly over space while the velocity $\vec{v}$ of the fluid is large, and such an approximation will not be valid in general, if we remember that (3.6) corresponds to a ground-state configuration. For instance, for the very simplest case of a non-interacting Bose gas, $\Psi(\vec{x})$ is simply constant over space (over a box in which the gas is confined) but the velocity $\vec{v}$ vanishes.

Phrased differently, a WKB approximation would correspond to a regime in which the fundamental degrees of freedom, i.e., the individual atoms in the case of the condensate or the degrees of freedom of quantum geometry in the case of GFT, already behave semiclassically. While semiclassical properties of the quantum state are an important consistency criterion in quantum cosmology (see, e.g., [92]), if quantum cosmology arises from the hydrodynamics of quantum gravity semiclassicality is not a restriction on the "wavefunction" $\sigma$ but rather a statement about the validity of an ansatz of the form (3.3), i.e., of the mean-field approximation. Early works on GFT condensates advocated the use of a WKB approximation [71, 72], but it was understood in $[67,69,70]$ that this is not really justified. In this review, we will focus on studies of the dynamics of GFT condensates that do not assume the validity of a WKB regime. Nevertheless, interesting results such as [43] where the dynamics of LQC and the effective dynamics of GFT condensates could be matched in a WKB regime show that the role of WKB solutions in this setting deserves to be better understood.

Because the "condensate wavefunction" is a mean-field configuration rather than a quantummechanical wavefunction, there is no obvious map between the formalism of GFT condensates and the Hilbert space formalism of Wheeler-DeWitt quantum cosmology or LQC, but such a map is not required; what is needed is a way to extract cosmological predictions from the effective dynamics of such condensates. For this it has proven advantageous to focus on expectation values of geometric operators that correspond to cosmological observables, as we will detail in Section 4.4 and below. Viewed more practically, the absence of a Hilbert space structure in this hydrodynamic setting might be seen as an advantage rather than a problem in quantum cosmology, where a general "wavefunction of the universe" has serious interpretational problems and one usually uses a class of special, highly semiclassical states, so that the full Hilbert space seems rather redundant when one is interested in the physical predictions of quantum cosmology. In our setting, the semiclassical properties expected from the universe are already encoded in the use of a restricted class of (condensate) states. 


\subsection{Interpretation in terms of geometry}

So far, the definition of GFT condensate states has mainly been motivated by the analogy with Bose-Einstein condensation in condensed matter physics. In order to relate this setting to quantum cosmology, we also need to show how states such as (3.3) or (3.4) can be interpreted in terms of spacetime geometry, i.e., in terms of (a probability distribution over) continuum metrics and/or Ashtekar-Barbero connections. For this, a slight detour is necessary in which we discuss in more detail the geometric interpretation of the configuration space of the GFT field $\hat{\varphi}\left(g_{I}\right)$ and, accordingly, the wavefunctions $\sigma$ and $\xi$ appearing in (3.3) and (3.4). Any interpretation proposed in this context is motivated by the close relation of GFT to LQG and spin foam models, which do give a geometric interpretation to the group-theoretic data associated to the states. Eventually, it will have to be shown that such an interpretation is justified, i.e., that the effective continuum geometry defined from it is governed by the dynamics of a gravitational theory (Einstein's equations, or a generalisation of them), couples correctly to matter, and satisfies a notion of diffeomorphism invariance. We will outline how this could be done for GFT condensates in the discussion of dynamics in Section 4.

What we are looking for is an "inverse map": given the parallel transports $g_{I}$ of a connection along four edges meeting at a central vertex,

$$
g_{I} \sim \mathcal{P} \exp \left(\int_{e_{I}} A\right)
$$

or, in the dual non-commutative formulation introduced in Section 2.3, four Lie algebra elements $B_{I}$ corresponding to bivectors associated to faces of a dual tetrahedron (where we assume that simplicity constraints are either trivial, for $G=\mathrm{SU}(2)$, or have been imposed properly),

$$
B_{I}^{A B} \sim \int_{\triangle_{I}} e^{A} \wedge e^{B}
$$

how do we reconstruct an (approximate) connection $A$ or a coframe field $e$ compatible with this data? This is a common problem in discrete approaches to quantum gravity, and hard to address $[38,39]$. Our task is somewhat easier than in general because we are looking at specific states with high symmetry, and expect the approximate continuum description to correspond to a homogeneous or almost homogeneous geometry.

The following interpretation of the bivectors (3.7) in terms of a continuum metric was given in [72]. First, the mean field $\sigma\left(g_{I}\right)^{6}$ has two independent symmetries $\sigma\left(g_{I}\right)=\sigma\left(h g_{I} k\right)$ for all $h, k \in G$, which imply that its noncommutative Fourier transform,

$$
\tilde{\sigma}\left(B_{I}\right):=\int \mathrm{d} g \prod_{I=1}^{4} e_{g_{I}}\left(B_{I}\right) \sigma\left(g_{I}\right)
$$

satisfies (compare with the property (2.8) of the GFT field itself)

$$
\tilde{\sigma}\left(B_{I}\right)=\delta_{\star}\left(\sum_{I} B_{I}\right) \star \tilde{\sigma}\left(B_{I}\right), \quad \tilde{\sigma}\left(B_{I}\right)=\tilde{\sigma}\left(k B_{I} k^{-1}\right) \quad \forall k \in G .
$$

These symmetries correspond to two symmetries in the continuum: the first is the closure constraint implying that the four faces close to form a geometric tetrahedron, and the second is the invariance under local frame rotations which are classical discrete gauge transformations.

\footnotetext{
${ }^{6}$ Here and in the following, $\sigma\left(g_{I}\right)$ stands for a general "condensate wavefunction" with the required symmetries, and in particular can also stand for the function $\xi\left(g_{I}\right)$ used in (3.4).
} 
The next step is to assume that the continuum fields to be reconstructed vary slowly over the scale of the tetrahedron, as must be the case for the discretisation to approximately represent them. This means we can replace $B_{i}^{A B}=\frac{l^{2}}{2} \epsilon_{i}{ }^{j k} e_{j}^{A} e_{k}^{B}$ in terms of three discrete triad vectors $e_{i}^{A}$, where $i=1,2,3$ and $l$ is a coordinate length associated to the triangles that we leave free for now; $B_{4}$ is replaced by $-\sum_{i} B_{i}$ using the closure constraint. The gauge-invariant configuration space for each tetrahedron, invariant under the transformations $B_{i} \mapsto k B_{i} k^{-1}$, is six-dimensional and can be parametrised by the six "metric components"

$$
g_{i j}=e_{i}^{A} e_{j}^{B} \eta_{A B}=\frac{1}{4 l^{2} \operatorname{tr}\left(B_{1} B_{2} B_{3}\right)} \epsilon_{i}^{k l} \epsilon_{j}^{m n} \tilde{B}_{k m} \tilde{B}_{l n}, \quad \tilde{B}_{i j}:=\operatorname{tr}\left(B_{i} B_{j}\right),
$$

where $\eta_{A B}$ is the appropriate invariant inner product. The quantities $g_{i j}$, constructed out of (3.7), should be scalars under diffeomorphisms. They represent the 3-metric in a given frame that transforms covariantly under diffeomorphisms, at a given fixed point in the tetrahedron (which can be chosen for convenience to be one of the vertices). The continuum interpretation of (3.9) is fixed after specifying this frame. In essence, the freedom in choosing this frame corresponds to the freedom in rotating tetrahedra in an embedding into a spatial manifold. This freedom is still there because our construction is purely local on the level of each individual tetrahedron and disregards any connectivity information in the states, as seems appropriate when considering the simple condensates of Section 3.1.

In order to define a notion of spatially homogeneous geometries, we now consider an embedding of a given configuration of tetrahedra into a continuum manifold of topology $\mathfrak{G} / X$ where $\mathfrak{G}$ is a 3-dimensional Lie group acting transitively on the manifold, and $X$ can be a discrete subgroup [72]. $\mathfrak{G}$ defines the notion of homogeneity, and also suggests a natural choice of frame, given by a basis of left-invariant vector fields. The $g_{i j}$ in (3.9) are then interpreted as metric coefficients in this frame, so that a spatially homogeneous geometry is precisely one in which $g_{i j}$ are all constant in the continuum, or the same for a large number of tetrahedra in the discrete. This then justifies the choice of state (3.1) or (3.3) in order to describe continuum spatially homogeneous geometries, where the continuum is strictly obtained in the limit $N \rightarrow \infty$.

Our use of an embedding in this context is very much analogous to how one interprets spin network states in canonical LQG as encoding (distributional) continuum geometric data on a given, continuum spatial hypersurface [124]. The topological information that one may associate to a collection of $N$ tetrahedra (i.e., a disjoint union of $N$ three-balls) plays no role in this continuum interpretation, and there would be no consistent way of using it if we are looking at superpositions of different $N$, e.g., (3.3). It seems plausible that, if cosmology and continuum geometry emerge from a hydrodynamic approximation to quantum gravity, the topology of space should also be emergent rather than determined by microscopic details, such a specific choice of graph. Indeed, the fact that one might think of a superfluid fundamentally as a collection of disconnected quantum atoms plays no role at the hydrodynamic level. This viewpoint leaves open the question of how to choose the embedding manifold, as we will discuss below. One might try to incorporate a choice of topology into the definition of the state: in Section 3.5, we will discuss a different class of condensates with definite topological interpretation, which may be seen as an alternative proposal.

In (3.9), we have focused on the metric which is usually more important in cosmology, but a similar construction of a continuum connection from the group elements $g_{I}$, taking into account the invariance under $g_{I} \mapsto h g_{I} k$, is possible. As discussed in some detail in [67] for $G=\mathrm{SU}(2)$, if GFT condensates define spatially homogeneous continuum geometries, the phase space of a tetrahedron can be mapped to the phase space of spatially homogeneous continuum geometries, with the quotient space $\mathrm{SU}(2) \backslash \mathrm{SU}(2)^{4} / \mathrm{SU}(2) \simeq \mathrm{SU}(2)^{3} / \mathrm{Ad}_{\mathrm{SU}(2)}$ of gauge-invariant group variables corresponding to continuum homogeneous connections and the quotient $\mathfrak{s u}(2)^{\oplus 3} / \mathrm{Ad}_{\mathrm{SU}(2)}$ of gauge-invariant bivector variables corresponding to continuum homogeneous metrics. This 


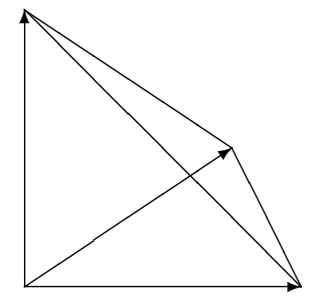

Figure 4. An "isotropic tetrahedron" with three pairwise orthogonal edges.

observation makes explicit in which sense a tetrahedron can be seen as a "chunk of space" with the same geometric data as a continuum geometry, and it provides an important foundation for the idea of cosmology as hydrodynamics of GFT: it shows that GFT condensate states, based on the idea of wavefunction homogeneity, capture exactly the degrees of freedom needed for homogeneous cosmology, i.e., minisuperspace geometric degrees of freedom. The ambiguities in interpreting these degrees of freedom are related to the ambiguity of defining an isomorphism between GFT data and continuum cosmological variables.

Using (3.9) gives a direct interpretation of the gauge-invariant geometric variables of a tetrahedron in terms of a continuum metric, which is relatively simple and local at the level of each tetrahedron. There are some technical difficulties in defining an operator version of (3.9), first from defining inverse operators, and also from operator ordering ambiguities coming from non-commutativity of the $\tilde{B}_{i j}$ [67]. Barring these, one could now define an effective (spatially homogeneous) continuum metric out of expectation values of such operators.

There are however several issues with (3.9), mainly related to its need to be supplemented by a choice of frame in which the $g_{i j}$ define the 3-metric. First of all, even though defining this frame by three left-invariant vector fields is rather natural, the notion of left-invariance itself relies on the choice of isometry group $\mathfrak{G}$, with the possible choices corresponding to the homogeneous cosmological models according to the Bianchi classification (see, e.g., [33]). The viewpoint taken in [72] is that this choice should be fixed by self-consistency: one does not know initially which of the Bianchi models is described by a particular condensate state, but its effective dynamics will pick out at most one, as the Bianchi models all differ by their "anisotropy potential" in the Hamiltonian constraint [33]. While this indeed suggests an unambiguous association of condensate states with Bianchi universes, a more fundamental understanding why a particular Bianchi geometry would be selected is so far lacking. Perhaps more importantly, the definition (3.9) uses only the bivectors $B_{1}, B_{2}$ and $B_{3}$, as $B_{4}$ has been eliminated using the closure constraint $\sum_{I} B_{I}=0$. The four faces of the tetrahedron are not treated "democratically": an isotropic tetrahedron would be one with three pairwise orthogonal edges of equal length, rather than a fully equilateral one, see Fig. 4. One might prefer constructions in which isotropy means equilateral tetrahedra [111]. The closure constraint also breaks the $\mathrm{O}(3)$ symmetry of continuum geometry under a redefinition of the basis of left-invariant vector fields; under such a redefinition, $e_{i} \mapsto O_{i}{ }^{j} e_{j}$ and $B_{i} \mapsto \pm O_{i}{ }^{j} B_{j}$ which in general does not preserve $\sum_{I} B_{I}=0$. This suggests that there should be a preferred basis of left-invariant vector fields, and for Bianchi models such a basis exists: it is the basis (defined up to the action of a permutation group $\mathfrak{S}_{3}$ ) in which the metric is diagonal, $g_{i j}=a_{i}^{2} \delta_{i j}$ with no summation over $i$. Hence, for consistency, we are required to assume that off-diagonal components of (3.9) vanish, at least as expectation values, which gives a restriction on possible condensate states to define Bianchi models. Again, this is not a problem in practice, but might suggest that the construction is not general enough ${ }^{7}$.

\footnotetext{
${ }^{7}$ It also appears that a similar issue would apply to connection variables, leading to the conclusion that these would also have to be diagonalised. Then one would be restricted to class A Bianchi models, for which a simultaneous diagonalisation of the spatial metric and its conjugate momentum is possible and a corresponding Hamiltonian formulation exists [33].
} 
So far, much of the work on GFT condensates has used geometric observables that do not rely on (3.9) but are built out of more basic operators borrowed from LQG (see Section 2.2). The perhaps most basic such operator is a volume operator, which in the second quantised formalism simply corresponds to the total volume contained in a state. The definition of the volume operator in LQG is somewhat subtle, with different definitions arising from different regularisation schemes [7, 126] and possible issues with a consistent semiclassical limit [65]; these issues are less important for tetrahedra, for which good agreement with Bohr-Sommerfeld quantisation has been found [29]. Given a definition of the volume operator, one can compute the expectation value or higher moments of the volume of the universe. For a homogeneous, isotropic geometry, this directly defines a scale factor $a$, proportional to the third root of this total volume. For more general geometries, the total volume is just the most coarse-grained geometric observable. Hence, for an identification of the total volume with a quantity proportional to $a^{3}$ to make sense, one needs to be confident that the state does define a homogeneous and isotropic geometry. The embedding picture above gives an argument for why condensates define spatially homogeneous geometries, but the notion of isotropy must come from elsewhere if one does not want to use the definition (3.9). For instance, one may define isotropy by equilateral tetrahedra, see Section 4.5 below.

As discussed in Section 2.2, the GFT Fock space formalism also allows the definition of "total area operators", one-body operators that are simpler to define than the total volume as they are directly built out of the basic flux operators in LQG. They take the form

$$
\widehat{A}_{I}=\int \mathrm{d} g \hat{\varphi}^{\dagger}\left(g_{J}\right) \sqrt{-\Delta_{g_{I}}} \hat{\varphi}\left(g_{J}\right)
$$

and describe quantities such as "the total area of all $I$-th faces". Their geometric interpretation is not entirely clear in general, since one seems to be summing over areas of disconnected surfaces, which is different from LQG operators that correspond to the area of a 2-surface in space. For a homogeneous and isotropic geometry, however, again the expectation value $\left\langle\widehat{A}_{I}\right\rangle$ of such an operator has to be proportional to the square of the scale factor. It turns out that $\left\langle\widehat{A}_{I}\right\rangle$ divided by $N^{1 / 3}$ can be identified with a cosmological scale factor, so that

$$
a^{2} V_{0}^{2 / 3}:=\left\langle\widehat{A}_{I}\right\rangle N^{-1 / 3}
$$

where $V_{0}$ is a fiducial coordinate volume, akin to the volume of the "fiducial cell" appearing in homogeneous models for quantum cosmology, such as LQC [11,32]. There are several arguments justifying the factor $N^{-1 / 3}$ in (3.10). First, consistency requires that the Poisson brackets of the effective cosmological quantities reduce to the ones of classical general relativity in the limit of low curvature [68], which fixes this factor. One can also motivate it heuristically by thinking of a large "fiducial cell" encompassing part of the universe one is interested in, itself composed of $N$ elementary cells. One would associate the cosmological area $a^{2} V_{0}^{2 / 3}$ to one of the sides of the fiducial cell. The operator $\widehat{A}_{I}$, however, takes into account all elementary cells, and hence overcounts by a factor $N^{1 / 3}$, the number of "layers" within the fiducial cell. Similar ideas have been used in LQC, where in order to connect lattice models and symmetry-reduced models one defines averages of fluxes over a lattice, rather than using a total flux (see, e.g., Section 3.6.1 in [34]). Also in that context, the number of layers to average over would be $N^{1 / 3}$. The factor $N^{1 / 3}$ is also consistent with a similar construction in [1], also rooted in LQC. All this suggests (3.10) as the most natural and useful definition of a cosmological scale factor $a$ from the "total area".

The study of inhomogeneities in GFT condensates is still in its infancy, and different ideas for their study have been put forward. One approach presumes that the quantum notion of wavefunction homogeneity does not necessarily lead to a universe that would be observed to be 
exactly homogeneous. Indeed, in any scenario which is not a truncation to homogeneity but an embedding of homogeneous geometries into a more general framework, one expects inhomogeneities to be present as quantum fluctuations, as restricting fluctuations over homogeneity to be exactly zero for both metric and connection would seem to violate the Heisenberg uncertainty relations. A prime example of this is inflation, where in the early universe quantum fluctuations undergo a transition to classical inhomogeneities, visible as temperature perturbations in the cosmic microwave background (CMB) today [89], in spite of the initial state being homogeneous. Put differently, condensate states have nonvanishing overlap with generic other states in the Fock space, in particular states that one would associate with generic inhomogeneous universes.

The mean-field approximation provides a direct way of implementing a transition from quantum to classical inhomogeneities for quantum geometry as represented by a GFT condensate [68]. Indeed, as discussed in Section 3.2, in this mean-field approximation the "condensate wavefunction" represents a classical field configuration - in the case of a real Bose-Einstein condensate it defines the density profile for a fluid in the laboratory. In this sense, in (3.3) the field $\sigma\left(g_{I}\right)$ represents a classical field configuration on the "connection minisuperspace" of group elements $g_{I}$, and its noncommutative Fourier transform $\tilde{\sigma}\left(B_{I}\right)$ (cf. (3.8)) a classical field on the "metric minisuperspace" of bivectors $B_{I}$. For any subset $C$ of such a minisuperspace, $\int_{C}|\sigma|^{2}$ counts the expected number of "patches" with metric/connection data in $C$; in this approximation, all "patches" have the same probability distribution of geometric data but they need not all be in exactly the same configuration when "measured" at a given moment. One distinguishes between wavefunction homogeneity as introduced in Section 3.1 and statistical, classical homogeneity, and is led to a picture of a "many-patch model" with a statistical distribution of inhomogeneities, just as is observed in the CMB, see Fig. 5. Some information about this statistical distribution can be extracted from expectation values of more complicated operators such as

$$
\widehat{\alpha}_{I}=\kappa^{2} \int \mathrm{d} g \hat{\varphi}^{\dagger}\left(g_{J}\right)\left(-\Delta_{g_{I}}\right) \hat{\varphi}\left(g_{J}\right),
$$

by comparing them with the area $\left\langle\widehat{A}_{I}\right\rangle$. As argued in [68], in the case of an isotropic geometry, and assuming a single scalar perturbation $\psi(\vec{x})$, one can approximately identify

$$
\int_{\mathbb{R}^{3}} \mathrm{~d} x|\psi(\vec{x})|^{2} \approx \frac{V_{0}}{4}\left(\frac{\left\langle\widehat{\alpha}_{I}\right\rangle N}{\left\langle\widehat{A}_{I}\right\rangle^{2}}-1\right),
$$

which reduces to zero in the exactly (quantum) homogeneous case where $\left\langle\widehat{\alpha}_{I}\right\rangle N=\left\langle\widehat{A}_{I}\right\rangle^{2}$.

Hence, even without any information about the embedding of the patches into space, limited information about perturbations can be extracted. The results of [68] outline the potential for a systematic analysis of classical inhomogeneities emerging from quantum fluctuations in the mean-field approximation. For more complicated condensate states, the precise relation is much less clear, and deserves further work.

An essentially orthogonal point of view, though not incompatible with the previous one, is that inhomogeneities should be included as fluctuations over the exact condensate, i.e., as phonons [72], or more simply as generalisations of the state (3.3) [69]. While looking at such perturbed condensates is no problem in principle, one faces the problem of interpreting the background-independent geometric data associated to the perturbation, given by group or Lie algebra elements, in terms of continuum fields on a manifold, where the manifold and the homogeneous background itself should be formed by the unperturbed condensate. One expects some similarities with the Born-Oppenheimer approximation in usual quantum cosmology (see, e.g., $[87,88])$ in the separation of degrees of freedom into homogeneous and inhomogeneous 


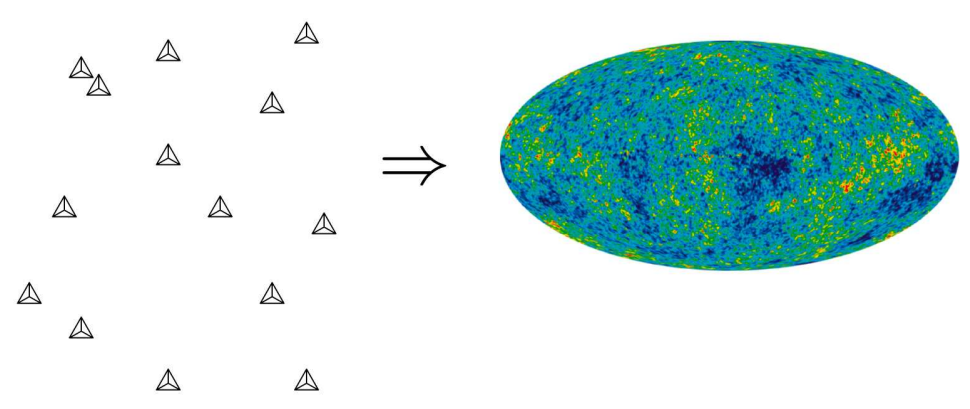

Figure 5. One of the main goals of research on GFT condensates.

parts, where the "background" is formed by a semiclassical wavefunction for the homogeneous mode. An interesting result of [69] was that the ratio of the number of quanta in the homogeneous background and in the fluctuation over it provides a natural small parameter not present in the continuum. However, what is missing so far is a notion of separating perturbation modes by "wavenumber", i.e., a Laplacian or spectral decomposition of modes with respect to the background geometry (presumably unrelated to the Laplacian on the group $G$ used in studies of GFT renormalisation [46, 47, 48]). The perturbations considered in [69] appear to be merely perturbations in the homogeneous mode, not qualitatively different from the unperturbed condensate.

\subsection{Discreteness and continuum limit}

The geometries defined by $N$-particle states in GFT or equivalently by a fixed graph in LQG are clearly discrete. In order for them to admit even an approximate continuum interpretation, $N$ must be very large, and the curvature should be very small when compared to the length scale of a tetrahedron. So far, there is no clear definition of a curvature operator in the GFT setting, which would be needed, through an analysis of its expectation values, to make this statement fully rigorous. In the more specific setting of condensate states, it is possible to still make this idea more precise by only referring to homogeneous degrees of freedom, as has been done in [72]; for instance, a near-flatness condition can be imposed by demanding that the average value of a function $\chi\left(g_{i} g_{4}^{-1} g_{j} g_{4}^{-1}\right)$, where $\chi$ is a character on the group, e.g., the trace in the $j=\frac{1}{2}$ representation for $G=\mathrm{SU}(2)$, is very close to $\chi(e)$ for all $i, j$. For a more detailed analysis, in a specific example, of condensate wavefunctions that correspond to such configurations of nearly flat tetrahedra, and hence admit an approximate continuum picture, see [67].

As we have mentioned, $N \rightarrow \infty$ corresponds to a continuum limit, which has to be understood as a phase transition, as it has been shown in great detail in the case of matrix models for twodimensional gravity [51]. For a phase transition to happen, the coupling constants of the GFT action will have to be tuned to critical values (if they exist) at which the expectation value of the number of quanta contained in the physical vacuum diverges. Phase transitions are only strictly possible for an infinite number of degrees of freedom, which means one is often mainly interested in the limit $N \rightarrow \infty$. Simultaneously, this "infinite volume" limit has to be accompanied by the divergence of correlation lengths of matter fields used to probe spacetime geometry. It is only in the continuum limit that symmetries such as diffeomorphism symmetry can be restored [54].

On the other hand, one of the central results of LQG has been the realisation that, at least at the kinematical level, the spectra of area and volume operators are discrete [126], although whether this implies discreteness for physical states is far from clear [58]. If there is a physical discreteness in the theory, we would be in a situation similar to causal sets [82] where there is no continuum limit but only a "continuum approximation". A GFT condensate describing a universe similar to ours would then have an extremely large but still finite number of degrees 
of freedom $N$, which for practical purposes may still be approximated as infinite.

The formalism of GFT condensates for cosmology, in the way it has been set up so far, essentially requires $N$ to be finite. First of all, this is because the limit $N \rightarrow \infty$ corresponds to a change of representation to a different Hilbert space, and while such a change may be highly desirable eventually to describe a phase transition, we are performing all calculations in the original Fock space around the perturbative GFT vacuum $\varphi=0$. Furthermore, quantities of cosmological interest such as the scale factor $a$ in (3.10) are defined using $N$, and become ill-defined in the $N \rightarrow \infty$ limit; we are defining approximate continuum quantities from the discrete variables of GFT, before any double-scaling limit of the usual type (discretisation scales go to zero, quantities of physical interest remain finite) is performed. In order to extend these constructions to the limiting case where the actual continuum limit is taken, a better understanding of this limit in GFT and the change in Fock space representation will be required. This said, it is also true that coherent states, even if they have a finite expectation value of the number operator, have nonvanishing projection over states with arbitrarily large number of quanta. Therefore, these approximate states are taking into account the idea of a continuum limit, even if in this limited sense. This is perhaps the most significant difference with respect to states built with a given number of degrees of freedom attached to a fixed graph.

Cosmologies based on a universe with a finite number of discrete degrees of freedom can have interesting properties of immediate phenomenological relevance. Perhaps the most striking is a proposal in the causal set approach [130], in which the smallness of the cosmological constant $\Lambda$ is conjectured to be a statistical effect arising from a very large but finite number of constituents of the universe. One expects fluctuations in $\Lambda$ to be of the order $\Lambda \sim 1 / \sqrt{N}$, and if $N$ is taken to be the number of Planck volumes in a Hubble volume this turns out to be roughly the observed value (this property is often considered part of the cosmological constant problem, the "coincidence problem"). While more of a general argument than a strict derivation, this idea shows how properties of the universe at the largest scales might depend on a fundamental discreteness of spacetime. More speculative ideas include attempts to derive deviations from scale invariance in the CMB power spectrum from fundamental discreteness [59].

The formalism of LQC is based on fundamental discreteness in an essential way. Indeed, its departures from the classical continuum Friedmann equation or Wheeler-DeWitt quantum cosmology arise from using the input of a non-zero minimal area from the kinematics of LQG $[11,32]$. In the derivation of the modifications to the continuum dynamics, the picture of a cosmological universe as defined on a large but fixed graph is used rather explicitly, e.g., in [9], without using a full LQG calculation. A crucial question in this context is how the (locally finite) number of degrees of freedom evolves with the expansion of the universe. The viewpoint emerging from [9] was that the volume of (a comoving portion of) the universe and the number of fundamental degrees of freedom should essentially be proportional, so that the volume per elementary cell is a constant (given by its minimal, Planckian value). More generally, LQC suggests a lattice refinement interpretation in which both quantities could evolve as the graph is refined in the evolution of the universe [36]. We will see later on (Section 4) that the picture of lattice refinement and the resulting form of holonomy corrections in LQC can be grounded in a natural way in the formalism of GFT condensates.

The picture of a graph to which new degrees of freedom are continuously added as the universe evolves leads to many open questions. One needs to ensure that any notion of homogeneity one wants to apply is preserved under such an evolution, which itself is fundamentally irreversible. For some work on this type of quantum dynamics, using Hilbert spaces of evolving dimension, see [83]. The open issue of how lattice refinement, as apparently required in LQC, is implemented dynamically in LQG provides additional motivation for the study of GFT condensates and their effective dynamics, which may provide a link between LQG and LQC by dynamically realising 
lattice refinement. Note that lattice refinement requires a dynamically changing number of degrees of freedom $N$, which indicates that working on a fixed graph in LQG may not be the appropriate setting for cosmology.

\subsection{Generalised condensates and generalisation to spherical symmetry}

As discussed in Section 3.1, the simplest condensate states of the form (3.3) or (3.4), while having attractive mathematical properties and a clear motivation by analogy with Bose-Einstein condensates, have shortcomings in their interpretation in terms of quantum geometry. In particular, we have seen that the absence of a connected graph means that there is no information about the topology of space, and that it is difficult to localise excitations, as seems required if one wants to explicitly add perturbations. This suggests defining generalised condensates on a nontrivial, connected graph. Here we review the essential ideas and technical points of this definition; full details can be found in [106].

Generalised condensates, just as the simple condensates of Section 3.1, are based on the idea of wavefunction homogeneity, i.e., the property of a many-particle state to be fully determined by a single-particle wavefunction. Denote this wavefunction again by $\sigma$ and define operators

$$
\hat{\sigma}\left(h_{I}\right)=\int \mathrm{d} g \sigma\left(h_{I} g_{I}\right) \hat{\varphi}\left(g_{I}\right), \quad \hat{\sigma}^{\dagger}\left(h_{I}\right)=\int \mathrm{d} g \bar{\sigma}\left(h_{I} g_{I}\right) \hat{\varphi}^{\dagger}\left(g_{I}\right) .
$$

Now fix a graph $\Gamma$ which encodes the desired topology, in the sense that it corresponds to the dual of a triangulation of a manifold of this topology, and define the "seed state"

$$
\mid \text { seed }\rangle \propto \int(\mathrm{d} h)^{\# V(\Gamma)} \prod_{e \in E(\Gamma)} \delta\left(h_{I}^{v}\left(h_{J}^{w}\right)^{-1}\right) \prod_{u \in V(\Gamma)} \hat{\sigma}^{\dagger}\left(h_{I}^{u}\right)|0\rangle,
$$

where $V(\Gamma)$ denotes the vertices in $\Gamma$ and $E(\Gamma)$ the edges of $\Gamma$; again we are using a slight shorthand notation where each edge $e$ is characterised as outgoing as the $I$-th link from the vertex $v$ and incoming as the $J$-th link into the vertex $w$.

The "seed state" is of the type often used in LQG, a state on a fixed graph, with a small number of degrees of freedom with respect to the Ashtekar-Lewandowski vacuum. To define from it a type of condensate representing some sort of continuum limit, and to incorporate a notion of coarse graining related to ideas such as [56], one now defines refinement operators which preserve the topology of the graph and the notion of wavefunction homogeneity. Both properties can be implemented by basing the refinement operators on Pachner moves [114], and by defining them using the wavefunction $\sigma$ that was already used for the seed state. For instance, in order to implement a $1 \rightarrow 4$ Pachner move one should find an operator $\widehat{\mathcal{M}}$ satisfying [106]

$$
\begin{aligned}
{\left[\widehat{\mathcal{M}}, \hat{\sigma}^{\dagger}\left(h_{1}, h_{2}, h_{3}, h_{4}\right)\right]=} & \int_{G^{6}} \mathrm{~d} g\left(\hat{\sigma}^{\dagger}\left(h_{4}, g_{1}, g_{2}, g_{3}\right) \hat{\sigma}^{\dagger}\left(g_{3}, h_{3}, g_{4}, g_{5}\right)\right. \\
& \left.\times \hat{\sigma}^{\dagger}\left(g_{5}, g_{2}, h_{2}, g_{6}\right) \hat{\sigma}^{\dagger}\left(g_{6}, g_{4}, g_{1}, h_{1}\right)\right),
\end{aligned}
$$

corresponding to the addition of three new vertices and six new edges to the graph; in this move, a tetrahedron is replaced by a more refined triangulation of a 3-ball, homeomorphic to the tetrahedron that was removed. Crucially, the action of $\widehat{\mathcal{M}}$ does not create disconnected components but only refines the given graph.

Once such an operator has been found, one can define generalised condensate states

$$
\left.\left|\Psi_{F}\right\rangle=F(\widehat{\mathcal{M}}) \mid \text { seed }\right\rangle
$$

for some function $F$. A natural choice motivated by technical simplicity and the analogy to the simple condensates of Section 3.1 would be an exponential. Even for this choice, expectation 
values for operators of geometric interest are quite difficult to compute. An interesting general result shown in [106] is that, for a certain class of refinement moves and initial seed states, expectation values of one-body operators of the general form

$$
\widehat{\mathcal{A}}=\int \mathrm{d} g \mathrm{~d} h A\left(g_{I}, h_{I}\right) \hat{\varphi}^{\dagger}\left(g_{I}\right) \hat{\varphi}\left(h_{I}\right)
$$

are extensive, i.e., reduce to a product of the average particle number $N=\langle\widehat{N}\rangle$ and a singleparticle expectation value given in terms of the wavefunction $\sigma$. This result is important for cosmology, where the scaling of observables with $N$ is a main property of interest.

The construction of generalised condensates leads in a rather straightforward way to a notion of spherical symmetry. The seed state can be chosen to correspond to a topology $S^{2} \times[0,1]$, interpreted as a spherical shell, where one needs to define different types of vertices that keep track of the inner boundary, outer boundary, and interior of the shell [106]. Gluing many such shells together using the inner boundary of one and the outer boundary of the next one, one obtains a more general configuration of spherical symmetry with nontrivial dependence along the radial direction, given by different wavefunctions $\sigma_{r}$ where $r$ labels the different shells.

For the construction of generalised condensates, it has proven advantageous to make use of tools developed in the context of coloured tensor models (see, e.g., [80, Section 4] for a concise presentation of the relevant procedures). The simple GFT models based on a single field are replaced with coloured models, possessing additional labels that facilitate the reconstruction of the topological structure of the boundary state, given the pattern of convolutions of the wavefunction. While the additional labels do not have an obvious counterpart in LQG, they are useful to deal with the problem of isolating the various contributions to quantities computed for families of different graphs. As the particular colouring introduced requires bipartite graphs, Pachner moves have to be replaced with melonic refinement moves. These refinement moves allow a better handling of the construction of a refined foliation, keeping fixed the topology. These small extensions are only required to make the construction easier to be dealt with in concrete cases, and do not change the substance of the general principles that we have discussed.

A first very interesting application of this formalism has been given in [107] where it was shown how to define a horizon entanglement entropy for such generalised condensates. There, thanks to the techniques developed in the second quantised formulation of GFT and of tensor models, it has been possible to get a first hint of the effect of superposing different graphs, a crucial aspect of the condensate picture, on a key physical quantity for semiclassical gravity, namely the entanglement entropy of subregions. It was shown that the entanglement entropy associated to an isolated horizon [52], defined in terms of GFT condensates, receives a (combinatorial) Boltzmann entropy contribution from the structure of the state in terms of its graph components, as the graph proliferation associated to the refinement moves involved has the correct scaling with the number of tetrahedra in the state. This feature of the generalised coherent states, in turn, gives rise to an area law with subleading logarithmic corrections, as expected from semiclassical gravity. This result already underscores the usefulness of GFT condensates and tensor model techniques for calculations that address the physics of many GFT degrees of freedom.

\section{GFT condensates: dynamics}

In Section 3, we described the construction of GFT condensate states at the kinematical level, and given their possible interpretation in terms of geometry and cosmology. The next step is to study the effective dynamics of such states, as extracted from the quantum dynamics of a given GFT model or class of GFT models. The restriction to a class of states such as (3.3) or (3.12) can be sufficient for obtaining manageable equations, but often further approximations are needed. Showing that there is a relation between the resulting effective dynamics and the 
dynamics of cosmological spacetimes in general relativity, such as the Friedmann equation for FRW backgrounds or dynamical equations for linearised perturbations, would not only give further support for the geometric interpretation of the abstract group-theoretic data appearing in GFT that we have put forward, but more importantly also give evidence that a certain GFT model, or class of models, can become serious contenders for a theory of quantum gravity in four dimensions. In this vein, one can hope to use GFT condensates to discriminate between different models, by restricting only to those that lead to an acceptable cosmological phenomenology. Ideally, a consistent picture of geometrogenesis would not only lead to a resolution of the Big Bang singularity in a consistent quantum theory, but also obviate the need for introducing inflationary models with their known theoretical problems [85].

As we will show in this section, the basic strategy for deriving effective dynamics for condensates from the fundamental GFT dynamics is rather straightforward, and in many ways analogous to how one proceeds in the case of Bose-Einstein condensates in condensed matter physics. We will give some detail on the resulting equations, while trying to be as general as possible, given the multitude of models in the GFT literature.

It has proven more difficult to identify interesting solutions to the resulting equations and to map them to an effective classical or semiclassical cosmological description. This is mainly because the resulting equations are almost always nonlinear and nonlocal with a peculiar type of nonlocality, and not many analytical solution techniques for such equations are known. Numerical techniques, while certainly a possible way forward, have not been employed much, and most of the work so far has focused on general and foundational aspects of the formalism.

\subsection{Gross-Pitaevskii equation as effective dynamics of a Bose condensate}

In the interest of making this review self-contained for readers who are not familiar with quantum field theory techniques in condensed matter physics, let us briefly review how the Gross-Pitaevskii equation, a certain type of "nonlinear Schrödinger equation", emerges from the effective dynamics of a quantum field theory for the atoms of a Bose-Einstein condensate.

In the simplest example, this quantum field theory can be defined by an action

$$
S[\Psi, \bar{\Psi}]=\int_{\mathbb{R}^{3} \times \mathbb{R}} \mathrm{d} x \mathrm{~d} t\left\{\frac{\mathrm{i} \hbar}{2}\left[\bar{\Psi} \frac{\partial \Psi}{\partial t}-\Psi \frac{\partial \bar{\Psi}}{\partial t}\right]-\left(\frac{\hbar^{2}}{2 m}|\vec{\nabla} \Psi|^{2}-\mu|\Psi|^{2}+V(\vec{x})|\Psi|^{2}+\frac{\lambda}{2}|\Psi|^{4}\right)\right\},
$$

where the scalar field $\Psi$ and its complex conjugate $\bar{\Psi}$, functions on non-relativistic flat spacetime $\mathbb{R}^{3} \times \mathbb{R}$, are conventionally treated as independent in the variation (this is basically equivalent to splitting the complex field $\Psi$ into two real components). Here $\mu$ represents a chemical potential and $\lambda$ is a coupling representing the (weak) interactions between atoms. We assume that the atoms have zero spin and that there is only a single species, but generalisations to spin degrees of freedom and to several species are straightforward. More importantly, it is assumed that the condensate is examined in a dilute limit, in which the interatomic distance is larger than the range of the interactions. This action should be seen as the first approximation of a more complicated theory which will include more terms describing all the possible interaction channels between the atoms. It is an effective field theory for the order parameter controlling a phase transition, whose regime of validity has to be checked a posteriori. However, it is also a generic prototype for a variety of different situations in which a weak coupling approximation, related to the low density of the fluid, can be applied.

Because the action is real, variations with respect to $\Psi$ and $\bar{\Psi}$ lead to only one independent complex equation, known as the Gross-Pitaevskii equation,

$$
\mathrm{i} \hbar \frac{\partial \Psi}{\partial t}=-\frac{\hbar^{2}}{2 m} \Delta \Psi-\mu \Psi+V(\vec{x}) \Psi+\lambda|\Psi|^{2} \Psi .
$$

This is the classical field equation for a complex field $\Psi$, nonlinear in $\Psi$ due to the last term. 
Proceeding with canonical quantisation, the first term in the action tells us that $\Psi$ and $\bar{\Psi}$ are canonically conjugate, and they should satisfy the Heisenberg algebra as operators, analogous to the GFT commutation relations (2.4). A Fock space is constructed in which $\hat{\Psi}(\vec{x})$ is a sum of annihilation operators and $\hat{\Psi}^{\dagger}(\vec{x})$ is a sum of creation operators with respect to a Fock vacuum $|0\rangle$. The excitations created by the ladder operators represent atoms.

The quantum dynamics given by the operator version of (4.1) is nonlinear and explicit solutions are hard to obtain in general. To proceed, one uses, in the simplest case, a mean-field approximation in which the operators are replaced by classical fields, i.e.,

$$
\hat{\Psi}(\vec{x})=\psi(\vec{x}) \mathbf{1}+\hat{\chi}(\vec{x}), \quad \hat{\Psi}^{\dagger}(\vec{x})=\bar{\psi}(\vec{x}) \mathbf{1}+\hat{\chi}^{\dagger}(\vec{x}),
$$

where one assumes that the expectation values of $\hat{\chi}$ and $\hat{\chi}^{\dagger}$ which represent fluctuations over the mean field $\psi$ and $\bar{\psi}$ are small and can be neglected in the simplest approximation. In such an approximation, the operator version of (4.1) evidently reduces again to the classical field equation (4.1) with $\Psi$ and $\bar{\Psi}$ replaced by $\psi$ and $\bar{\psi}$.

To make this more precise, we would like to justify the mean-field approximation in the Fock space formalism by constructing a state satisfying $\hat{\Psi}(\vec{x})|\psi\rangle=\psi(\vec{x})|\psi\rangle$. This property is satisfied by the coherent state

$$
|\psi\rangle \propto \exp \left(\int_{\mathbb{R}^{3}} \mathrm{~d} x \psi(\vec{x}) \hat{\Psi}^{\dagger}(\vec{x})\right)|0\rangle .
$$

The similarity to our construction (3.3) for GFT should be obvious. It is also clear that there is no such state that also satisfies $\hat{\Psi}^{\dagger}(\vec{x})|\psi\rangle=\bar{\psi}(\vec{x})|\psi\rangle$, which would be in contradiction with the fact that $\hat{\Psi}$ and $\hat{\Psi}^{\dagger}$ do not commute. Hence the mean-field approximation can only correspond to an expectation value of the (normal ordered) operator equations of motion;

$$
\left\langle\psi\left|\frac{\delta S\left[\hat{\Psi}, \hat{\Psi}^{\dagger}\right]}{\delta \hat{\Psi}^{\dagger}}\right| \psi\right\rangle=\left\langle\psi\left|\mathrm{i} \hbar \frac{\partial \hat{\Psi}}{\partial t}+\frac{\hbar^{2}}{2 m} \Delta \hat{\Psi}+\mu \hat{\Psi}-V(\vec{x}) \hat{\Psi}-\lambda \hat{\Psi}^{\dagger} \hat{\Psi}^{\dagger} \hat{\Psi}\right| \psi\right\rangle=0
$$

is equivalent to the Gross-Pitaevskii equation for the field $\psi$.

We repeat that, for the general nonlinear Gross-Pitaevskii equation, there is no consistent interpretation of $\psi(\vec{x})$ as an actual single-particle wavefunction; there is no probability interpretation attached to it, as one has already taken an expectation value, and there is no Hilbert space based on such "wavefunctions" which are essentially parameters for a certain class of states in the Hilbert space of the theory, the non-relativistic Fock space. Rather, this wavefunction includes hydrodynamic information, as it can be shown using the Madelung representation (cf. (3.6)) $\psi(\vec{x})=\sqrt{\rho(\vec{x})} \exp (-\mathrm{i} \theta(\vec{x}))$. The modulus of the wavefunction controls the number density, while the phase controls the velocity through the velocity potential $\theta$, such that $\vec{v}=\frac{\hbar}{m} \vec{\nabla} \theta$. See Section 3.2 in the kinematical part for an extended discussion of this point, and its meaning for the "nonlinear quantum cosmology" derived from the effective dynamics of GFT condensates.

When interactions can be ignored $(\lambda=0)$, a single-particle wavefunction solving the (then standard) Schrödinger equation appears as the function $\psi(\vec{x})$ in the mean-field approximation; the latter is then simply the statement that one has a large number of identical non-interacting bosonic particles in a certain potential.

The main lesson from the well-understood case of condensed matter physics for our treatment of the effective GFT dynamics is the importance of approximations not only in the choice of state but also in the truncation of the full dynamics to expectation values such as (4.2). Indeed, in much of the following we will explore the hypothesis that the analogue of (4.2), in the case of GFT, provides a good approximation to the full quantum GFT dynamics. 


\subsection{Effective equations from GFT Schwinger-Dyson equations}

Given that GFTs, as introduced in Section 2, are quantum field theories with a structure rather similar to the non-relativistic field theories used in condensed matter physics, the most straightforward way of obtaining an effective dynamics for GFT condensates is from expectation values similar to (4.2). The simplest choice is indeed

$$
\left\langle\sigma\left|\frac{\delta S\left[\hat{\varphi}, \hat{\varphi}^{\dagger}\right]}{\delta \hat{\varphi}^{\dagger}\left(g_{I}\right)}\right| \sigma\right\rangle=0
$$

where $S$ is now the action of a given GFT model and $\hat{\varphi}$ and $\hat{\varphi}^{\dagger}$ are the field operators introduced in Section 2. For the simplest choice of condensate state (3.3), which implements the mean-field approximation, this then directly gives the GFT analogue of the Gross-Pitaevskii equation: the mean field $\sigma\left(g_{I}\right)$ should satisfy the classical GFT equations of motion,

$$
\int \mathrm{d} h K\left(g_{I}, h_{I}\right) \sigma\left(h_{I}\right)+\frac{\delta V[\sigma, \bar{\sigma}]}{\delta \bar{\sigma}\left(g_{I}\right)}=0 .
$$

This equation is now not only nonlinear but also nonlocal in $\sigma$, even for a local kinetic operator $K$, due to the nonlocal combinatorial pairing of group arguments in the interaction part $V$. This suggests that exact solutions will not be easily obtained.

Already for the "dipole condensate" (3.4) and certainly for the generalised condensates (3.12), the expectation value (4.3) is much harder to obtain. In general one will not find a closed expression in terms of the "condensate wavefunction" (also denoted $\sigma$ in the general case) without further approximations, such as an expansion in powers of $\sigma$ that is truncated at some order (see, e.g., [129]). Certain choices for $K$ and $V$ can lead to models that can be solved more directly.

One may also be interested in going beyond the truncation to only a single expectation value (4.3). Indeed, (4.3) can be seen as the simplest of an infinite tower of Schwinger-Dyson equations for the GFT model in question. Formally, Schwinger-Dyson equations can be derived from assuming a "fundamental theorem of functional calculus" by which, schematically,

$$
0=\int \mathcal{D} \varphi \mathcal{D} \bar{\varphi} \frac{\delta}{\delta \bar{\varphi}\left(g_{I}\right)}\left(\mathcal{O}[\varphi, \bar{\varphi}] e^{-S[\varphi, \bar{\varphi}]}\right)=\left\langle\frac{\delta \mathcal{O}[\varphi, \bar{\varphi}]}{\delta \bar{\varphi}\left(g_{I}\right)}-\mathcal{O}[\varphi, \bar{\varphi}] \frac{\delta S[\varphi, \bar{\varphi}]}{\delta \bar{\varphi}\left(g_{I}\right)}\right\rangle
$$

holds in the vacuum state for any sufficiently well-behaved functional $\mathcal{O}$ of the field and its complex conjugate (such as a polynomial). Since we are looking for condensates that can play the role of a new, non-perturbative vacuum of the theory, one can impose (4.5), for some choices of $\mathcal{O}$, as consistency conditions relating different $n$-point functions, which have to be satisfied in a physical condensate state. Using Schwinger-Dyson equations to extract an effective dynamics of GFT condensates was first proposed in [71, 72].

In practice, beyond the truncation imposed by considering a few very simple choices for $\mathcal{O}$, so far another important approximation has been made: $n$-point correlation functions are computed as expectation values in the inner product of the Fock space, i.e., of the Hilbert space of a "free" GFT. One could try to go beyond this approximation using methods such as used in [113], where general closed expressions for two-point functions were derived using Schwinger-Dyson equations and then solved in a perturbative expansion in the GFT coupling constant(s).

\subsection{Effective equations from projection methods}

So far we have proceeded via field-theoretic considerations. We want to briefly mention further possibilities to define an effective dynamics from the microscopic theory, introducing some form of coarse graining. In many condensed matter physics problems, in the majority of the cases 
where the exact ground state is not known, the objective is to find the best approximation for the properties of the ground state of a system, while keeping track of a reasonable amount of observables. In GFT we do not have a proper notion of energy to minimise, but it is still possible to adapt many of the techniques used in condensed matter physics. In doing so, one can really appreciate the power of the second quantised formulation that we are using.

The ultimate objective of the GFT programme as we have presented it is to provide predictions for physical observables, even in a simplified context as the one of cosmological dynamics. This means that the final results will be specific relations between the observables of relevant physical quantities (in our case, scale factors, Hubble parameter, etc.), as is also a necessity in a background-independent language as the one of GFT. The spirit of condensate states that we are advocating is clear: they are a way to obtain such a coarse-grained picture by identifying a simple set of variables (contained implicitly in the condensate wavefunction) and restricting their values with some equations of motion appropriately derived from the microscopic theory.

It is natural to ask whether one can bypass the condensate idea and work out the relations between observables directly. A possibility, proposed in [129], is to adapt the projection methods used in statistical mechanics to derive the equations of motion of selected observables from the (quantum) Liouville equation for the whole system [14, 42].

Instead of working with a pure state, we will have to work with a (generically mixed) density matrix adapted to the relevant observables $\mathfrak{A}=\left\{\hat{A}_{i}, i=1, \ldots, N\right\}$, which is designed using a maximum entropy principle. The idea is that, at fixed (but unspecified) values of the expectation values $\mathcal{A}_{i}$ of the observables in $\mathfrak{A}$, the density matrix that maximises the von Neumann entropy will have the general shape

$$
\hat{\rho}(\gamma)=\exp \left(\Psi(\gamma)-\gamma^{i} \hat{A}_{i}\right)
$$

where $\gamma^{i}$ are the chemical potentials, dual to the observables, and $\Psi$ is the logarithm of the partition function, up to a sign. The chemical potentials are determined implicitly by

$$
\mathcal{A}_{i}=\frac{\partial \Psi}{\partial \gamma^{i}}
$$

as usual. The idea is to determine the values of the chemical potentials such that the density matrix that they identify can be used in place of the exact microscopic state, at least as far as the operators in $\mathfrak{A}$ are concerned.

The problem, then, is to find the values of $\gamma$ that best reflect the microscopic equation of motion. In the GFT setting, we do not have notions such as time evolution and a ground state of minimal energy from which we can construct approximation methods (such as, for instance, the variational method in time-independent quantum-mechanical problems). A natural option is to require that the overlap between the reduced density matrix and a microscopic solution of the equations of motion is maximal. This simple idea is based on the notion of fidelity, which has been used within the context of quantum phase transitions [135] to explore their dynamical aspects. This reasoning leads immediately to a variational principle for the observables, which follows from maximising the overlap between the approximate density matrix and the density matrix of the exact state $|\Phi\rangle\langle\Phi|$

$$
\exp (\Sigma(\gamma))=\operatorname{Tr}(\hat{\rho}(\gamma)|\Phi\rangle\langle\Phi|)
$$

The effective equations are obtained varying this action with respect to the chemical potentials, and finding its maxima. The solutions will then lead, via (4.6), to the desired expectation values that best match the physical state. Additionally, by looking at fluctuations around the mean values, one can provide a first assessment of the validity of the given approximation, which is one of the crucial aspects when giving predictions for physical observables. 
It goes without saying that these expressions are rather formal, and they require appropriate regularisation in order to be used concretely, especially given the possibility that the physical state might not be a normalisable state in the Fock space. Their usefulness is in the more explicit presentation of a path to be followed in developing a thermodynamic approach to GFT, where only macroscopic quantities directly relevant for physics are used, as well as a direct inclusion of a maximum entropy (or maximum ignorance) principle in the derivation of the effective equations. As we mentioned for the case of generalised condensates, we should expect that the equations for the observables will be very complicated, requiring further simplifications before being reduced to tractable problems. This is of course not a problem faced by GFT alone.

Interestingly, this discussion can be used to motivate, from a different perspective, the GrossPitaevskii equation for GFT condensates. Indeed, for a simple form of the quantum equations of motion (trivial kinetic term and interaction containing only creation operators), one can show that the exact quantum state solving the equations of motion has a very simple, albeit formal, representation of the form of the generalised condensate states,

$$
|\Phi\rangle \sim \exp \left(-V\left[\hat{\varphi}^{\dagger}\right]\right)|0\rangle
$$

Such an expression is still formal and does not display clearly the geometric properties of the state. Therefore, even if the exact solution is available, we still need some approximation to extract the information it contains, i.e., we need a state whose properties we can easily understand and that provides the best approximation for the exact state. If we consider then the problem of maximising the fidelity of a coherent state on the physical state, along the lines that we have just discussed, we obtain exactly the desired Gross-Pitaevskii equation [129].

Besides putting the discussion of the GFT condensates under a different light, this point of view shows how GFT can contribute to the concrete realisation of some of the ideas often considered in emergent gravity scenarios [128] in a fully background-independent context.

\subsection{Dynamics of cosmological observables}

Once effective dynamical equations for GFT condensates have been obtained, from SchwingerDyson equations or by projection methods, one needs to connect them to cosmology. This connection is made by identifying cosmological observables as expectation values of GFT Fock space operators, as we have discussed in Section 3.3. In the simplest case, one is interested in observables that can be constructed out of local variables at the level of each tetrahedron, such as the "total areas"

$$
A_{I}=\left\langle\widehat{A}_{I}\right\rangle:=\kappa\left\langle\int \mathrm{d} g \hat{\varphi}^{\dagger}\left(g_{J}\right) \sqrt{-\Delta_{g_{I}}} \hat{\varphi}\left(g_{J}\right)\right\rangle
$$

or the effective metric components (3.9). It is also possible to construct a notion of curvature that is local for each tetrahedron, as mentioned in Section 3.4. By construction, any quantities of this type, arising as expectation values of one-body operators of the form

$$
\widehat{X}=\int \mathrm{d} g \mathrm{~d} g^{\prime} X\left(g_{I}, g_{I}^{\prime}\right) \hat{\varphi}^{\dagger}\left(g_{I}\right) \hat{\varphi}\left(g_{I}^{\prime}\right)
$$

are extensive, i.e., scale with the number of quanta $N$. In order to obtain observables that have the correct large-scale geometric interpretation, expectation values of one-body operators (4.8) must be rescaled with appropriate powers of $N$; for instance, in order to have a notion of curvature, expectation values of one-body operators (4.8) must be rescaled with $1 / N$ [70].

Effective dynamical equations can then be translated into equations for the dynamics of observables. To clarify this procedure, let us detail a simple example, building on an observation 
first made in [71]: choosing $\mathcal{O}=\varphi\left(g_{I}^{\prime}\right)$ in (4.5) leads to the consistency condition

$$
\int \mathrm{d} h K\left(g_{I}, h_{I}\right)\left\langle\hat{\varphi}\left(g_{I}^{\prime}\right) \hat{\varphi}\left(h_{I}\right)\right\rangle+\left\langle: \frac{\delta V\left[\hat{\varphi}, \hat{\varphi}^{\dagger}\right]}{\delta \hat{\varphi}^{\dagger}\left(g_{I}\right)} \hat{\varphi}\left(g_{I}^{\prime}\right):\right\rangle=0,
$$

where we are expressing expectation values in the operator formalism using normal ordering, i.e., the second expectation value is to be taken after normal ordering. An equivalent equation holds for $\mathcal{O}=\bar{\varphi}\left(g_{I}^{\prime}\right)$ as there is no contribution from $\delta \bar{\varphi} / \delta \bar{\varphi}$ which is zero after normal ordering (it is a delta distribution and hence equal to a fundamental commutator).

One can now assume the dipole condensate state (3.4) in which all odd $n$-point functions vanish, as is straightforward to verify. Then, in a GFT model of the form (2.1) corresponding to one of the usual spin foam models in four dimensions, where the potential is built out of $\varphi^{5}$ terms, the second term in (4.9) only contains odd $n$-point functions and is therefore zero, meaning we are left with two equations for two-point functions,

$$
\int \mathrm{d} h K\left(g_{I}, h_{I}\right)\left\langle\xi\left|\hat{\varphi}^{\dagger}\left(g_{I}^{\prime}\right) \hat{\varphi}\left(h_{I}\right)\right| \xi\right\rangle=\int \mathrm{d} h K\left(g_{I}, h_{I}\right)\left\langle\xi\left|\hat{\varphi}\left(g_{I}^{\prime}\right) \hat{\varphi}\left(h_{I}\right)\right| \xi\right\rangle=0 .
$$

Thus, in this special case, only the two-point functions of the condensate state and only the "kinetic" (quadratic) part of the GFT dynamics contribute to the effective dynamics. This is clearly a drastic approximation, due to the specific choice of state (3.4), and only the simplest of an in principle infinite tower of consistency conditions arising from (4.5). It would suggest that the precise form of the potential $V$ is not relevant in the effective description, as long as it is built out of terms with odd powers in the GFT field. Nevertheless, we can use (4.10) as a starting point to understand the more general procedure. One can motivate (4.10) without using the specific dipole condensate state (3.4), by working in a weak-coupling limit of the GFT in which contributions of the interaction terms may be neglected on more general grounds.

Further specifying to a kinetic term that is local, $K\left(g_{I}, h_{I}\right)=\delta\left(g_{I}^{-1} h_{I}\right) \mathcal{D}_{g}$ where $\mathcal{D}$ can be a differential operator, setting $g_{I}^{\prime}=g_{I}$ and integrating, the first equation of (4.10) yields

$$
\int \mathrm{d} g\left\langle\xi\left|\hat{\varphi}^{\dagger}\left(g_{I}\right) \mathcal{D}_{g} \hat{\varphi}\left(g_{I}\right)\right| \xi\right\rangle=0,
$$

which is now of the form of an expectation value for a one-body operator, specified by $\mathcal{D}_{g}$, which can be translated into cosmological observables. This very direct route from SchwingerDyson equations to cosmological observables, which avoids the need for discussing an effective Wheeler-DeWitt equation, was first used in [70].

In the case $\mathcal{D}_{g} \propto \mathbf{1}$, as is often the starting point for GFT models corresponding to spin foams, (4.11) simply gives $N=0$, i.e., the only solution is the Fock vacuum; for nontrivial solutions to arise, the operator $\mathcal{D}_{g}$ should have a nontrivial kernel. Work in GFT renormalisation $[46,47,48]$ suggests that the kinetic term must contain a Laplace-Beltrami operator on the group in order to allow a separation of scales (given by the eigenvalues of this Laplacian), and be of the form

$$
\mathcal{D}_{g}=-\sum_{I} \Delta_{g_{I}}-\mu^{2}
$$

where $\Delta_{g_{I}}$ is a Laplace-Beltrami operator acting on the $I$-th argument, and $\mu^{2}$ is a (dimensionless) coupling constant specifying the relative weight between the two terms. With (4.12), the interpretation of (4.11) given in [68] was, for an exactly homogeneous and isotropic universe,

$$
a^{3} V_{0}=\left(\frac{\kappa \mu}{2}\right)^{3 / 2} N
$$

where $a$ is the cosmological scale factor, $V_{0}$ is a fiducial coordinate volume (so that $a^{3} V_{0}$ corresponds to the physical volume), and $\kappa$ is the "Planck area" of the model. This is a nontrivial 
result, as it suggests that the physical volume should be proportional to the number of degrees of freedom, as seems required to reproduce the improved dynamics scheme of LQC [9] (see also [36, 43, 70] for related discussions of this connection, in particular [43] for an alternative derivation of LQC improved dynamics from GFT). There are corrections to (4.13) if inhomogeneities are present, see [68] for details.

A recent idea that makes a more direct connection to quantum cosmology has been the introduction of relational cosmological observables in [111]. Working in a model in which a massless scalar field $\phi$ is introduced explicitly as an argument of the GFT field,

$$
\hat{\varphi}\left(g_{I}\right) \rightarrow \hat{\varphi}\left(g_{I}, \phi\right),
$$

one can define quantities like the number operator at a fixed value of $\phi$,

$$
\hat{N}\left(\phi_{0}\right)=\int \mathrm{d} g \hat{\varphi}^{\dagger}\left(g_{I}, \phi_{0}\right) \hat{\varphi}\left(g_{I}, \phi_{0}\right),
$$

where $\phi$ plays, as usual in quantum cosmology, the role of a relational clock [134]. In a similar way, one can introduce an operator $\hat{V}\left(\phi_{0}\right)$ for the total volume of the universe at a fixed value of the scalar field, which is one of the main relational observables of interest in LQC [11, 32]. One can then obtain an effective Friedmann equation as a relation between $V(\phi) \equiv\langle\hat{V}(\phi)\rangle$ and its derivatives with respect to $\phi$, and show how it reduces to the Friedmann equations for general relativity and LQC in certain cases; see Section 4.5 for a summary of these most recent, particularly promising results.

\subsection{Effective Friedmann equations and relation to LQC}

One of the main goals of the study of GFT condensates has been the derivation of an effective quantum cosmology dynamics that can be compared with the results of minisuperspace models such as LQC. As discussed in Section 3.2, such a comparison cannot happen directly at the level of Wheeler-DeWitt-like equations, given that "condensate wavefunctions" do not play the role of "wavefunctions of the universe". While early work such as [71, 72] tried to interpret equations such as (4.10) directly as wave equations for a quantum cosmology wavefunction and accordingly applied approximation methods such as a WKB approximation to their study, this viewpoint has now been replaced by the study of expectation values that can be associated with cosmological observables. The results obtained by these two approaches are related, given that cosmological observables are often extensive so that there is an appropriate scaling with the number of quanta $N$ that relates the "single-particle wavefunction" and "condensate wavefunction" viewpoints; there is no discussion of this scaling in [71, 72] which was better understood in [70].

The appearance of $N$, which has no analogue in standard quantum cosmology or in a continuum setting, can allow a derivation of properties of effective quantum cosmology models from the more fundamental setting of GFT. For example, as shown in [70], the form of holonomy corrections of LQC can be obtained from the appropriate scaling with $N$ in the cosmological interpretation of GFT observables: parametrising $\mathrm{SU}(2)$ group elements by the coordinates

$$
g=: \sqrt{1-|\vec{\pi}[g]|^{2}} \mathbf{1}-\mathrm{i} \vec{\sigma} \cdot \vec{\pi}[g], \quad|\vec{\pi}[g]| \leq 1,
$$

if such a group element is identified, for example, with a parallel transport along an edge of coordinate length $l$ in the $x$-direction, we have

$$
g=: \mathcal{P} \exp \int_{e} \omega \approx \exp \left(l \omega_{x}\right) \leadsto \vec{\pi}[g]=-\vec{\omega}_{x} \sin \left(l\left|\vec{\omega}_{x}\right|\right) /\left|\vec{\omega}_{x}\right|
$$

where we use $\mathfrak{s u}(2) \simeq \mathbb{R}^{3}$ to view the connection $\omega$ (assumed to be constant over this edge) as an element of $\mathbb{R}^{3}$. Note that the introduction of a coordinate system led to the replacement of 
the coordinate-independent expression $\int_{e} \omega$ by $l \omega_{x}$ where $\omega=\omega_{x} d x+\cdots$. If we then define a macroscopic "total group element" operator by

$$
\widehat{\Pi_{i}^{2}}:=\int \mathrm{d} g\left(\vec{\pi}\left[g_{i} g_{4}^{-1}\right]\right)^{2} \hat{\varphi}^{\dagger}\left(g_{I}\right) \hat{\varphi}\left(g_{I}\right),
$$

the results of $[68,70]$ show that $l \sim N^{-1 / 3}$, and thus

$$
\left\langle\widehat{\Pi_{i}^{2}}\right\rangle=: N \sin ^{2}\left(\left(V_{0} / N\right)^{1 / 3}\left|\vec{\omega}_{i}\right|\right)
$$

defines an effective macroscopic connection variable $\omega$. This result shows how the fundamental group variables are related to the sine of the connection multiplied by a function of $N$ that determines the type of holonomy corrections in LQC [11, 32]. In particular, the (tentative) result (4.13) would suggest that this function of $N$ is proportional to the inverse scale factor, which precisely defines the improved dynamics scheme in LQC [9]. The demonstration that the form of LQC holonomy corrections can be obtained from considerations in GFT has been a major achievement of the work on GFT condensates. Note that it requires no assumptions about the specific form of the dynamics, as it is done essentially at the kinematical level.

While such kinematical results are very promising, one would also like to match the effective dynamics arising from GFT condensates to a type of Friedmann equation coming from classical general relativity (or a modified classical theory of gravity) or from a quantum gravity-inspired setting such as LQC. As we have explained, the most promising approach towards this seems to be to compute the dynamics of cosmological observables as expectation values of operators on the GFT Fock space, and to compare the resulting equations with classical or semiclassical dynamical equations for the corresponding quantities. In the setting of $\mathrm{LQC}$, this means focusing on effective equations that approximate the dynamics for appropriate semiclassical states (see, e.g., [32]), rather than the full quantum formalism in terms of a Hilbert space and corresponding Wheeler-DeWitt equation. Again, this is consistent with the condensate viewpoint, which suggests that one should work with a special class of semiclassical states, rather than the highly non-classical states given by generic elements of a quantum cosmology Hilbert space. The results of $[111,112]$ show how such an effective Friedmann equation, (4.16), can be extracted from GFT condensates; they follow previous work in this GFT condensate setting, in which various derivations of effective "Friedmann" equations have been discussed [43, 68, 70, 71, 72]. These derivations use different approximations which need to be independently justified, and more work is needed on extracting such equations that in particular use the structure of the GFT interactions, which have been largely neglected so far.

Let us give some more detail on the recent results of $[111,112]$, which form the most complete derivation of such generalised Friedmann equations from GFT condensates so far. One focuses on the relational observable $V(\phi)$, giving the volume of a comoving part of the universe (the "fiducial cell") at a fixed value of a scalar field $\phi$ that plays the role of relational clock. In classical general relativity, $V(\phi)$ satisfies the equations

$$
\left(\frac{1}{3 V} \frac{d V}{d \phi}\right)^{2}=\frac{4 \pi G_{\mathrm{N}}}{3}, \quad \frac{1}{V} \frac{d^{2} V}{d \phi^{2}}=12 \pi G_{\mathrm{N}}
$$

Note that the right-hand side is equal to a constant in both cases. The first equation gets modified in LQC where a term proportional to the matter density $\rho$ appears,

$$
\left(\frac{1}{3 V} \frac{d V}{d \phi}\right)^{2}=\frac{4 \pi G_{\mathrm{N}}}{3}\left(1-\frac{\rho}{\rho_{c}}\right),
$$

where $\rho_{c}$ is a critical (Planckian) density. In [111], the expectation values $V(\phi), V^{\prime}(\phi)$ and $V^{\prime \prime}(\phi)$ are computed for a condensate state of the form (3.3), with an additional argument $\phi$ of the 
GFT field and the condensate wavefunction representing the physical scalar field; the mean field $\sigma\left(g_{I}, \phi\right)$ is restricted to a state of isotropic (equilateral) tetrahedra,

$$
\sigma\left(g_{I}, \phi\right)=\sum_{j=0}^{\infty} \sigma_{j}(\phi) \mathbf{D}^{j}\left(g_{I}\right),
$$

where $\mathbf{D}^{j}\left(g_{I}\right)$ is an appropriate (fixed) convolution of Wigner $D$-matrices with SU(2) intertwiners implementing isotropy, such that the only geometric degree of freedom left in the condensate wavefunction $\sigma$ is the representation label $j$. One then uses the equations of motion (4.4) for an extension of the EPRL model [62]; in terms of the variable $j$ these take the form

$$
A_{j} \partial_{\phi}^{2} \sigma_{j}(\phi)-B_{j} \sigma_{j}(\phi)+w_{j} \bar{\sigma}_{j}(\phi)^{4}=0,
$$

where $A_{j}, B_{j}$ and $w_{j}$ are parameters specified on one hand by the details of the GFT action and on the other hand by the intertwiners contained in the functions $\mathbf{D}^{j}\left(g_{I}\right)$, associated to the specific choices operated in the selection of the isotropic degrees of freedom. Note that (4.17) decouples different $j$ components, which can hence be studied separately. This is a direct consequence of the particular form of the spin foam vertex chosen for the EPRL model, which possesses a specific pattern of identification of the spins. Other models, for instance the one proposed in [17], will not necessarily have this decoupling property and therefore will lead to different evolution equations and, possibly, different physical predictions.

In the cases in which the decoupling of the different spins occurs, the system of equations (4.17) can be easily studied with conventional methods used for the study of classical dynamical systems: the problem boils down to determining the behaviour of a system of noninteracting particles in two dimensions (since the wavefunction is complex) subject to the action of an external potential, whose precise shape is determined by the GFT interaction term. Consequently, it is possible to determine, at least with qualitative methods, the properties of the full family of the solutions to the complete nonlinear equations, in the isotropic restriction, and to study their dependence on the parameters $A_{j}, B_{j}$ and $w_{j}$.

In the derivation of the analogue of (4.15) and (4.16), one now uses (4.17) where interactions are neglected $\left(w_{j} \rightarrow 0\right)$, so that the only relevant feature of the amplitude that is required is the plausible restriction that the spin labels of faces of the tetrahedra in the boundary of the four-simplex, when glued, are in fact the same. (4.17) includes a nontrivial kinetic operator $K$ due to the coupling to the scalar field, to get the corresponding Klein-Gordon kinetic term in the GFT amplitudes.

The main result of $[111,112]$ is to show several cases in which, for appropriate choices of the couplings, the function $V(\phi)$ indeed satisfies (4.15), and to give the leading corrections. One finds that the effective Newton's constant $G_{N}$ is given in terms of the couplings $A_{j}$ and $B_{j}$, and hence originates in the structure of the GFT action. Interestingly, for a condensate in which all tetrahedra are constrained to have the same fixed volume (given by a single spin $j_{0}$ ), exactly a correction of the improved dynamics LQC form (4.16) is found, together with additional quantum corrections. This shows how GFT condensates can be governed by dynamics that are consistent not only with general relativity but also with LQC. Morever, the correct form of improved dynamics corrections arises without the need to introduce operators for the connection variables and argue for the dependence of holonomy corrections on $N$, as in (4.14), thus strengthening the connection between LQC improved dynamics and the more fundamental setting of GFT.

Several assumptions have to be made in order to obtain these results, see [111] for details. The most significant approximation is that, as in previous calculations, the GFT interaction term has to be neglected when matching the effective dynamics to (4.15). As the magnitude of the mean field $\sigma\left(g_{I}\right)$ is related to the number of quanta, interactions become more and more relevant as 
the expectation value of $\widehat{N}\left(\phi_{0}\right)$ becomes large, so that this approximation becomes less justified. For the possible (polynomial) interaction terms that must be presumably added to the simplicial interaction term to take care of radiative corrections, higher monomials have a higher impact on the effective dynamics. As the coefficients of these terms are not known, in absence of strong arguments from consistency of the quantum theory, we would be facing a problem of non-universality of the macroscopic dynamics. This is not a signal that GFT models do not reproduce general relativity in a large volume limit, but rather that the simple condensate state approximation (3.3) is not appropriate: as can be seen from the quantum equations of motion, as the number of tetrahedra increases, the coherent state deviates more and more from the exact state. The coherent state picture seems valid for a limited mesoscopic regime in which there is the first formation of a GFT condensate. After the first stages, correlations between different quanta will not be negligible anymore and it will be necessary to replace simple coherent states with more complex states. This is not incompatible with the idea of GFT condensation, as these more complicated states can still be controlled by cosmological wavefunctions, and, e.g., be of the generalised form discussed in Section 3.5. More work is needed to see whether general relativity can be recovered from the dynamics of these more complicated states.

As we mentioned in the introduction, nonlinear dynamical equations for quantum cosmology can also be motivated, independently of any assumptions on fundamental quantum gravity, as modelling inhomogeneities by interactions between different locally homogeneous patches. A concrete model of this type was studied in [37], leading to a nonlinear, nonlocal extension of quantum cosmology; a more general class of models, serving as nonlinear extensions of LQC, had been given, with similar motivations, in [44].

The construction of such models follows the general structure of building a quantum field theory in "second quantisation", where the kinetic operator in the quadratic term of the action corresponds to the wave equation satisfied by a single-particle wavefunction. Starting from homogeneous, isotropic LQC defined in terms of a volume variable $\nu$ and a massless scalar field $\phi$, this leads to a general action of the form [44]

$$
S[\Psi]=\frac{1}{2} \sum_{\nu} \int_{\mathbb{R}} \mathrm{d} \phi \Psi(\nu, \phi) \hat{\mathcal{K}} \Psi(\nu, \phi)+\sum_{j=2}^{n} \frac{\lambda_{j}}{j !} \sum_{\nu_{1} \ldots \nu_{j}} \int_{\mathbb{R}^{j}} \mathrm{~d} \phi f_{j}\left(\nu_{i}, \phi_{i}\right) \prod_{k=1}^{j} \Psi\left(\nu_{k}, \phi_{k}\right),
$$

where $\hat{\mathcal{K}}$ corresponds to the Hamiltonian constraint operator of a chosen LQC model,

$$
\hat{\mathcal{K}} \Psi(\nu, \phi):=-B(\nu)\left(\Theta+\partial_{\phi}^{2}\right) \Psi(\nu, \phi),
$$

where $\Theta$ is a difference operator only acting on the variable $\nu$ (which takes discrete values, $\nu \in \nu_{0} \mathbb{Z}$ ) and $B(\nu)$ is a given function; for some examples from LQC, see [44]. The interaction kernels $f_{j}$ should be chosen to model inhomogeneities, as made more concrete in [37].

One could hope to obtain (4.18) or a similar form as an effective action for GFT condensates, where $\Psi$ either corresponds to a specific condensate wavefunction (for a homogeneous, isotropic universe) or to a perturbation of the GFT field around a background field, as proposed in a similar context in [94], depending on the interpretation of the "second quantised" scalar field $\Psi$ in (4.18). Such a result would show that a Hamiltonian constraint derived directly from general relativity captures the effective dynamics of GFT, and thus give evidence that the corresponding GFT model does indeed define a theory of quantum gravity. It is not necessary that such a direct mapping should exist: what is really needed is evidence that the collective behaviour of many degrees of freedom, described by large-scale observables, behaves as predicted by general relativity. Obtaining such observables from the fundamental GFT variables presumably requires coarse-graining over many orders of magnitude (in the simplest case, rescaling by some power of $N$ ), and it may not be possible to find an even approximate description of the macroscopic 
variables in terms of an effective "LQC wavefunction". Nevertheless, it is interesting to study the behaviour of nonlinear quantum cosmology models such as (4.18) in their own right, if only as toy models for the effective dynamics of GFT condensates. For instance, a linear but nonlocal extension of the Wheeler-DeWitt equation was recently studied in [49].

In order to extract cosmological phenomenology from the effective dynamics of GFT condensates, it is clearly necessary to have matter in the model. Depending on how realistic a cosmology one wants to describe, this could for example be a radiation fluid, a scalar field modelling inflation, or a massless scalar field that can serve as a relational clock [134]. While most GFT models aim to define pure quantum gravity without matter, there are also models $[66,108]$ in which matter fields are introduced explicitly, by enlarging the number of microscopic degrees of freedom associated to each basic building block. Alternatively, it has been suggested that matter degrees of freedom might already be included in the excitations of GFT degrees of freedom over a background $[63,75]$ in which case there would be no need to add them explicitly.

Both approaches can be followed in the cosmological setting of GFT condensates. The first approach leads to an extension of the domain space of the GFT field and the GFT action. This was first done in the context of the GFT condensates in [72], where it was shown how the simplest extension to a scalar field led to the correct matter coupling to gravity for a massless scalar field in the effective Friedmann equation. More recently, the more systematic constructions of [111] give further support to this approach, by explicitly showing how the Friedmann equations of classical general relativity and their LQC corrections can arise from the dynamics of an appropriate condensate state and GFT model.

In the second approach, one tries to interpret corrections to an effective Friedmann equation (arising, for instance, from different types of GFT interactions) as matter fields or as an effective cosmological constant. This possibility was outlined in [70] where we point for further reference. It is potentially very interesting, as it may, for example, raise a hope to solve the cosmological constant problem through GFT condensates, but has not been explored much so far.

\section{Main results, open questions and future directions}

We close by summarising the main results, some of the remaining open questions and some directions for current and future research.

The first major achievement [71, 72] was the development of a formalism for the definition of group field theory condensates and for their geometric interpretation; the procedure outlined in Section 3.3 showed how an LQG "graph" consisting of a large number $N$ of disconnected elementary building blocks (open spin network vertices or gauge-invariant "dipoles") can be interpreted as a macroscopic, approximately smooth homogeneous geometry, if one imposes the criterion of wavefunction homogeneity that forces all building blocks to be in the same single-particle configuration. The definition (3.9) of an approximate continuum metric from geometric data in $N$-particle states was new and rather simple compared to other proposals in the literature. As we have described in Section 3.3, some issues related to it need to be resolved, in particular as it only seems consistent in a frame in which the spatial metric is diagonal. The second main result of $[71,72]$ was to show how an effective dynamics could be extracted from GFT Schwinger-Dyson equations, and reinterpreted as an effective quantum cosmology model. Agreement with the vacuum Friedmann equation of Lorentzian and Riemannian general relativity, in a WKB approximation, was found, and it was also shown how to include a massless scalar field, leading to the correct coupling of the scalar field to gravity in the effective Friedmann equation. Similar techniques were subsequently applied in [43] leading to a successful derivation of the LQC improved dynamics scheme from this GFT model coupled to matter.

Further work [67, 69, 68, 70] clarified some of the conceptual and technical aspects of the framework. First, in [67] analytical solutions to the "Wheeler-DeWitt equation" of $[71,72]$ were 
analysed in more detail and it was found that there are no WKB-like solutions satisfying the requirements of [71, 72]: highly oscillating "condensate wavefunctions" do not correspond to nearly flat tetrahedra and hence do not describe an approximate continuum (see Section 3.4). This already suggested that using the WKB approximation may not be justified. These results were followed up by [70] where it was shown how the relation between cosmological observables and observables on the GFT Fock space involves a nontrivial scaling with the particle number $N$ (see, e.g., (3.10)), which is not present in standard quantum cosmology. This allowed a derivation of the form of LQC holonomy corrections in [70], depending on the unknown relation $N=N(a)$ between number of degrees of freedom $N$ and scale factor $a$, similar to the lattice-refinement picture of LQC $[34,36]$. It was also understood how the WKB approximation applied in the earlier works corresponds to a presumably unphysical regime in which individual "atoms of space" behave semiclassically; put differently, it corresponds to a limit of $l_{\text {Planck }} \rightarrow 0$ at fixed $N$, not the expected continuum limit which would be of double-scaling form $l_{\text {Planck }} \rightarrow 0$ and $N \rightarrow \infty$. As a consequence, the "Friedmann equations" given in [70] contain additional terms compared to $[71,72]$ that come with extra powers of both $l_{\text {Planck }}$ and $N$. It was suggested that these terms could act as effective matter components in the effective Friedmann equation.

Semiclassicality, a crucial issue in quantum cosmology where the emergence of a classical universe needs to be explained [92], was discussed in more detail in [68]: the mean-field approximation, encoded in the choice of state (3.3) and a large number of quanta $N$, already corresponds to a semiclassical limit; the mean-field configuration $\sigma\left(g_{I}\right)$ represents a classical GFT field configuration rather than a first-quantised quantum cosmology wavefunction. Consequently, it should be interpreted as defining a "patch density" on a minisuperspace of geometric data, given by the parallel transports $g_{I}$ or area bivectors $B_{I}$ - the hydrodynamic description of a GFT condensate corresponds to a "fluid" on such a minisuperspace, or a statistical distribution of patches with locally different geometries [68]. This interpretation implements a quantum-toclassical transition exactly of the type needed in inflation, where the quantum fluctuations of the inflaton field over a homogeneous, isotropic vacuum state lead to classical statistical inhomogeneities and anisotropies. A conceptually rather similar idea, akin to the hydrodynamic picture of a Bose-Einstein condensate, was in fact recently proposed in [2] to solve this "measurement problem" of inflation. The formalism of GFT condensates here connects directly to some of the main conceptual questions in inflation, and suggests that inhomogeneities can arise from a non-zero spread of the density profile on minisuperspace [68].

The conceptual basis of the framework, and the map between the fundamental GFT dynamics and the effective description in terms of cosmology, are now rather well understood. What remains is to apply these ideas to concrete GFT models of interest, constructing appropriate, physically interesting condensate states. So far, it has proven rather difficult to include the GFT interaction terms into the analysis, and indeed almost all of the existing work has neglected them, either in a weak-coupling limit or by choosing specific states and SchwingerDyson equations that are unaffected by the interactions. This is ultimately unsatisfactory, as results obtained from such calculations would not be sensitive to most of the details of the GFT or spin foam model used, and the approximation one makes appears not to be justified as the number of GFT quanta becomes large. In the construction of states, recently [106, 107] more emphasis has been put on constructing a more general class of condensate states that allow the use of a nontrivial LQG graph structure and its associated topology, and also generalise the setting from spatial homogeneity to spherical symmetry, opening an avenue for exploring black holes and their thermodynamics: indeed it has been shown [107] that an appropriately defined horizon entanglement entropy for such generalised condensates gives an area law with subleading logarithmic corrections. The cosmological phenomenology of these generalised condensates has not been explored much, as the resulting effective dynamical equations will be more involved, and additional approximation techniques presumably need to be developed to handle them. 
A main question that this approach faces is whether its effective dynamics, at some semiclassical level, can reduce to the effective dynamics of LQC, or more generally to general relativity up to corrections at high curvature. Here, we can list the kinematical results of [70] that derived the general form of holonomy corrections as functions of $N$, and the more concrete calculations of $[43,68]$ that gave evidence for the improved dynamics form of these holonomy corrections. A derivation of LQC inverse-triad corrections [11,32] would presumably require a clearer understanding of the definition of inverse volume operators in the GFT setting. Dynamically, the clearest and promising results that show a derivation of modified Friedmann equations, of the precise LQC form, from the dynamics of GFT condensates have been obtained in [111, 112], assuming a certain matter coupling in GFT, a type of semiclassical limit, and neglecting GFT interactions (see Section 4.5 for further discussion). The latest results in particular show that such a derivation of LQC dynamics is possible and may be rather universal, given that only specification of the kinetic term seems required. They allow explicit contact with LQC and beyond, as one can also compute interesting modifications to general relativity and LQC, which characterise different GFT models and allow further distinguishing between them.

Further progress will depend on the development of better approximation techniques, in order to handle more complicated condensate states, and include more information about the GFT dynamics. Numerical techniques, which have so far hardly been used (see, however, [119]), will become useful in the more detailed study of various GFT models. Apart from this, most other open questions address the validity of the GFT condensate picture itself. The approximations implied by assuming that a certain type of condensate state is a good approximation to a physical state of the full theory need to be quantified and verified in detail. To some extent, one can rely on insight from related situations in condensed matter physics, where the types of states we have considered describe a weakly interacting, diluted Bose-Einstein condensate, and one can see where this approximation breaks down by looking at the effect of interactions and by computing correlation functions [118]. In the GFT context, similar calculations should be performed, which connect to results on the phase structure and renormalisation of GFT models (see Section 1.2). As we have said, one would like to understand the breakdown of the condensate approximation in terms of a phase transition, and relate it to the scenario of "geometrogenesis" where the geometric phase given by a GFT condensate was preceded by a different, non-geometric phase.

Again on a more technical level, the reconstruction procedure of Section 3.3 needs to be refined and understood better in the setting of simplicial geometry and LQG; more generally, a clearer definition of isotropic and anisotropic geometries, in terms of a detailed mapping to the possible Bianchi geometries, needs to be developed, in order to extend the existing calculations for isotropic models to anisotropies. For inhomogeneities, in Section 3.3 we have mentioned one way of identifying them in the mean-field approximation of the simplest condensates, but there may be clearer ways of including inhomogeneities, allowing their localisation on the emergent space given by the GFT condensate. Indeed, the main obstacle to a derivation of a power spectrum of inhomogeneities in a GFT condensate seems to be the absence of a good notion of "wavenumber" (usually defined through the spectrum of a suitable Laplace operator). The existing results of $[69,68]$ are not fully conclusive, since they only describe homogeneous perturbations or global quantities such as (3.11) characterising the inhomogeneities. The ideas of [72] for including inhomogeneities as a type of phonons, while quite natural from the condensate perspective, have not been explored in much detail. In many ways, a clearer treatment of inhomogeneities, both technically and conceptually, in GFT condensates is one of the key open issues, given that the availability of detailed observations of the CMB power spectrum would offer a very concrete way to put a whole class of quantum gravity models to a direct observational test.

A general problem faced by the GFT/LQG community in order to obtain more realistic models is how matter is to be included into models of interest. GFT condensates allow approaching 
it in the same way as for pure gravity models: given a proposal for including matter, one can define the appropriate condensate states, compute the effective dynamics, and check that they reduce to the classical Friedmann equation coupled to matter, perhaps up to quantum corrections. We have shown one such calculation, in the EPRL model coupled to a massless scalar field, in Section 4.5, and the promising results should motivate further work on similar models.

To conclude, group field theory condensates have already been shown to provide very generally applicable methods for extracting an effective description of the collective behaviour of a large number of degrees of freedom of quantum geometry, defined by a GFT. Techniques and approximation methods for obtaining and solving effective equations can, to some extent, be borrowed from condensed matter physics, but need to be modified to the peculiar structure (in particular, the combinatorial type of nonlocality) of GFT models. Developing such methods further seems to be the main step missing for a full derivation of cosmological phenomenology of GFT, including GFT interactions. Several results, in particular those of $[111,112]$, already indicate a close relation of the resulting semiclassical dynamics to effective equations in LQC.

\section{Acknowledgements}

We thank Martin Bojowald, Daniele Oriti, Andreas Pithis and Edward Wilson-Ewing and the anonymous referees for helpful comments on the manuscript. The research leading to these results has received funding from the People Programme (Marie Curie Actions) of the European Union's Seventh Framework Programme (FP7/2007-2013) under REA grant agreement $\mathrm{n}^{\mathrm{o}} 622339$ and from the John Templeton Foundation through the grant PS-GRAV/1401.

\section{References}

[1] Alesci E., Cianfrani F., Loop quantum cosmology from quantum reduced loop gravity, Europhys. Lett. 111 (2015), 40002, 5 pages, arXiv:1410.4788.

[2] Alexander S., Jyoti D., Magueijo J., Inflation and the quantum measurement problem, Phys. Rev. D 94 (2016), 043502, 5 pages, arXiv:1602.01216.

[3] Alexandrov S., Geiller M., Noui K., Spin foams and canonical quantization, SIGMA 8 (2012), 055, 79 pages, arXiv:1112.1961.

[4] Ambjørn J., Görlich A., Jurkiewicz J., Loll R., Nonperturbative quantum gravity, Phys. Rep. 519 (2012), 127-210, arXiv:1203.3591.

[5] Ashtekar A., New variables for classical and quantum gravity, Phys. Rev. Lett. 57 (1986), $2244-2247$.

[6] Ashtekar A., Lewandowski J., Representation theory of analytic holonomy $C^{*}$-algebras, in Knots and Quantum Gravity (Riverside, CA, 1993), Oxford Lecture Ser. Math. Appl., Vol. 1, Oxford University Press, New York, 1994, 21-61, gr-qc/9311010.

[7] Ashtekar A., Lewandowski J., Quantum theory of geometry. II. Volume operators, Adv. Theor. Math. Phys. 1 (1997), 388-429, gr-qc/9711031.

[8] Ashtekar A., Lewandowski J., Background independent quantum gravity: a status report, Classical Quantum Gravity 21 (2004), R53-R152, gr-qc/0404018.

[9] Ashtekar A., Pawlowski T., Singh P., Quantum nature of the big bang: improved dynamics, Phys. Rev. D 74 (2006), 084003, 23 pages, gr-qc/0607039.

[10] Ashtekar A., Rovelli C., Smolin L., Weaving a classical metric with quantum threads, Phys. Rev. Lett. 69 (1992), 237-240, hep-th/9203079.

[11] Ashtekar A., Singh P., Loop quantum cosmology: a status report, Classical Quantum Gravity 28 (2011), 213001, 122 pages, arXiv:1108.0893.

[12] Baez J.C., An introduction to spin foam models of BF theory and quantum gravity, in Geometry and Quantum Physics (Schladming, 1999), Lecture Notes in Phys., Vol. 543, Springer, Berlin, 2000, 25-93, gr-qc/9905087.

[13] Bahr B., Dittrich B., Geiller M., A new realization of quantum geometry, arXiv:1506.08571. 
[14] Balian R., Incomplete descriptions and relevant entropies, Amer. J. Phys. 67 (1999), 1078-1090, cond-mat/9907015.

[15] Baratin A., Dittrich B., Oriti D., Tambornino J., Non-commutative flux representation for loop quantum gravity, Classical Quantum Gravity 28 (2011), 175011, 19 pages, arXiv:1004.3450.

[16] Baratin A., Oriti D., Group field theory with noncommutative metric variables, Phys. Rev. Lett. 105 (2010), 221302, 4 pages, arXiv:1002.4723.

[17] Baratin A., Oriti D., Group field theory and simplicial gravity path integrals: a model for Holst-Plebanski gravity, Phys. Rev. D $\mathbf{8 5}$ (2012), 044003, 15 pages, arXiv:1111.5842.

[18] Baratin A., Oriti D., Ten questions on group field theory (and their tentative answers), J. Phys. Conf. Ser. 360 (2012), 012002, 10 pages, arXiv:1112.3270.

[19] Barbero G. J.F., Real Ashtekar variables for Lorentzian signature space-times, Phys. Rev. D 51 (1995), 5507-5510, gr-qc/9410014.

[20] Barbieri A., Quantum tetrahedra and simplicial spin networks, Nuclear Phys. B 518 (1998), 714-728, gr-qc/9707010.

[21] Barceló C., Liberati S., Visser M., Analogue gravity, Living Rev. Relativ. 14 (2011), 3, 159 pages, gr-qc/0505065.

[22] Barrett J.W., Dowdall R.J., Fairbairn W.J., Gomes H., Hellmann F., Asymptotic analysis of the Engle-Pereira-Rovelli-Livine four-simplex amplitude, J. Math. Phys. 50 (2009), 112504, 30 pages, arXiv:0902.1170.

[23] Ben Geloun J., Renormalizable models in rank $d \geq 2$ tensorial group field theory, Comm. Math. Phys. 332 (2014), 117-188, arXiv:1306.1201.

[24] Ben Geloun J., Gurau R., Rivasseau V., EPRL/FK group field theory, Europhys. Lett. 92 (2010), 60008, 6 pages, arXiv:1008.0354.

[25] Ben Geloun J., Martini R., Oriti D., Functional renormalization group analysis of a tensorial group field theory on $\mathbb{R}^{3}$, Europhys. Lett. 112 (2015), 31001, 6 pages, arXiv:1508.01855.

[26] Ben Geloun J., Martini R., Oriti D., Functional renormalization group analysis of tensorial group field theories on $\mathbb{R}^{d}$, Phys. Rev. D 94 (2016), 024017, 45 pages, arXiv:1601.08211.

[27] Benedetti D., Ben Geloun J., Oriti D., Functional renormalisation group approach for tensorial group field theory: a rank-3 model, J. High Energy Phys. 2015 (2015), no. 3, 084, 40 pages, arXiv:1411.3180.

[28] Bianchi E., Doná P., Speziale S., Polyhedra in loop quantum gravity, Phys. Rev. D 83 (2011), 044035, 17 pages, arXiv:1009.3402.

[29] Bianchi E., Haggard H.M., Discreteness of the volume of space from Bohr-Sommerfeld quantization, Phys. Rev. Lett. 107 (2011), 011301, 4 pages, arXiv:1102.5439.

[30] Bianchi E., Rovelli C., Vidotto F., Towards spinfoam cosmology, Phys. Rev. D 82 (2010), 084035, 8 pages, arXiv:1003.3483.

[31] Bodendorfer N., Quantum reduction to Bianchi I models in loop quantum gravity, Phys. Rev. D 91 (2015), 081502, 6 pages, arXiv:1410.5608.

[32] Bojowald M., Loop quantum cosmology, Living Rev. Relativity 11 (2008), 4, 131 pages, gr-qc/0601085.

[33] Bojowald M., Canonical gravity and applications: cosmology, black holes, and quantum gravity, Cambridge University Press, Cambridge, 2010.

[34] Bojowald M., Mathematical structure of loop quantum cosmology: homogeneous models, SIGMA 9 (2013), 082, 43 pages, arXiv:1206.6088.

[35] Bojowald M., Quantum cosmology: a review, Rep. Progr. Phys. 78 (2015), 023901, 21 pages, arXiv:1501.04899.

[36] Bojowald M., Cartin D., Khanna G., Lattice refining loop quantum cosmology, anisotropic models, and stability, Phys. Rev. D 76 (2007), 064018, 13 pages, arXiv:0704.1137.

[37] Bojowald M., Chinchilli A.L., Simpson D., Dantas C.C., Jaffe M., Nonlinear (loop) quantum cosmology, Phys. Rev. D 86 (2012), 124027, 13 pages, arXiv:1210.8138.

[38] Bombelli L., Corichi A., Winkler O., Semiclassical quantum gravity: statistics of combinatorial Riemannian geometries, Ann. Phys. 14 (2005), 499-519, gr-qc/0409006.

[39] Bombelli L., Corichi A., Winkler O., Semiclassical quantum gravity: obtaining manifolds from graphs, Classical Quantum Gravity 26 (2009), 245012, 15 pages, arXiv:0905.3492. 
[40] Bonzom V., Gurau R., Ryan J.P., Tanasa A., The double scaling limit of random tensor models, J. High Energy Phys. 2014 (2014), no. 9, 051, 49 pages, arXiv:1404.7517.

[41] Boulatov D.V., A model of three-dimensional lattice gravity, Modern Phys. Lett. A 7 (1992), 1629-1646, hep-th/9202074.

[42] Breuer H.P., Petruccione F., The theory of open quantum systems, Oxford University Press, New York, 2002.

[43] Calcagni G., Loop quantum cosmology from group field theory, Phys. Rev. D 90 (2014), 064047, 13 pages, arXiv:1407.8166.

[44] Calcagni G., Gielen S., Oriti D., Group field cosmology: a cosmological field theory of quantum geometry, Classical Quantum Gravity 29 (2012), 105005, 22 pages, arXiv:1201.4151.

[45] Calcagni G., Oriti D., Thürigen J., Dimensional flow in discrete quantum geometries, Phys. Rev. D 91 (2015), 084047, 11 pages, arXiv:1412.8390.

[46] Carrozza S., Group field theory in dimension $4-\varepsilon$, Phys. Rev. D 91 (2015), 065023, 10 pages, arXiv:1411.5385.

[47] Carrozza S., Oriti D., Rivasseau V., Renormalization of tensorial group field theories: Abelian U(1) models in four dimensions, Comm. Math. Phys. 327 (2014), 603-641, arXiv:1207.6734.

[48] Carrozza S., Oriti D., Rivasseau V., Renormalization of a SU(2) tensorial group field theory in three dimensions, Comm. Math. Phys. 330 (2014), 581-637, arXiv:1303.6772.

[49] de Cesare M., Gargiulo M.V., Sakellariadou M., Semiclassical solutions of generalized Wheeler-DeWitt cosmology, Phys. Rev. D 93 (2016), 024046, 15 pages, arXiv:1509.05728.

[50] De Pietri R., Freidel L., Krasnov K., Rovelli C., Barrett-Crane model from a Boulatov-Ooguri field theory over a homogeneous space, Nuclear Phys. B 574 (2000), 785-806, hep-th/9907154.

[51] Di Francesco P., Ginsparg P., Zinn-Justin J., 2D gravity and random matrices, Phys. Rep. 254 (1995), 1-133, hep-th/9306153.

[52] Diaz-Polo J., Pranzetti D., Isolated horizons and black hole entropy in loop quantum gravity, SIGMA 8 (2012), 048, 58 pages, arXiv:1112.0291.

[53] Dittrich B., From the discrete to the continuous: towards a cylindrically consistent dynamics, New J. Phys. 14 (2012), 123004, 27 pages, arXiv:1205.6127.

[54] Dittrich B., The continuum limit of loop quantum gravity - a framework for solving the theory, arXiv:1409.1450.

[55] Dittrich B., Geiller M., A new vacuum for loop quantum gravity, Classical Quantum Gravity 32 (2015), 112001, 13 pages, arXiv:1401.6441.

[56] Dittrich B., Martin-Benito M., Steinhaus S., Quantum group spin nets: refinement limit and relation to spin foams, Phys. Rev. D 90 (2014), 024058, 15 pages, arXiv:1312.0905.

[57] Dittrich B., Steinhaus S., Time evolution as refining, coarse graining and entangling, New J. Phys. 16 (2014), 123041, 42 pages, arXiv:1311.7565.

[58] Dittrich B., Thiemann T., Are the spectra of geometrical operators in loop quantum gravity really discrete?, J. Math. Phys. 50 (2009), 012503, 11 pages, arXiv:0708.1721.

[59] Dreyer O., The world is discrete, arXiv:1307.6169.

[60] Dupuis M., Livine E.R., Lifting SU(2) spin networks to projected spin networks, Phys. Rev. D 82 (2010), 064044, 11 pages, arXiv:1008.4093.

[61] Engle J., Embedding loop quantum cosmology without piecewise linearity, Classical Quantum Gravity 30 (2013), 085001, 14 pages, arXiv:1301.6210.

[62] Engle J., Livine E., Pereira R., Rovelli C., LQG vertex with finite Immirzi parameter, Nuclear Phys. B 799 (2008), 136-149, arXiv:0711.0146.

[63] Fairbairn W.J., Livine E.R., 3D spinfoam quantum gravity: matter as a phase of the group field theory, Classical Quantum Gravity 24 (2007), 5277-5297, gr-qc/0702125.

[64] Fleischhack C., Kinematical foundations of loop quantum cosmology, arXiv:1505.04400.

[65] Flori C., Thiemann T., Semiclassical analysis of the loop quantum gravity volume operator. I. Flux coherent states, arXiv:0812.1537.

[66] Freidel L., Oriti D., Ryan J., A group field theory for 3d quantum gravity coupled to a scalar field, gr-qc/0506067. 
[67] Gielen S., Quantum cosmology of (loop) quantum gravity condensates: an example, Classical Quantum Gravity 31 (2014), 155009, 20 pages, arXiv:1404.2944.

[68] Gielen S., Identifying cosmological perturbations in group field theory condensates, J. High Energy Phys. 2015 (2015), no. 8, 010, 23 pages, arXiv:1505.07479.

[69] Gielen S., Perturbing a quantum gravity condensate, Phys. Rev. D 91 (2015), 043526, 11 pages, arXiv:1411.1077.

[70] Gielen S., Oriti D., Quantum cosmology from quantum gravity condensates: cosmological variables and lattice-refined dynamics, New J. Phys. 16 (2014), 123004, 11 pages, arXiv:1407.8167.

[71] Gielen S., Oriti D., Sindoni L., Cosmology from group field theory formalism for quantum gravity, Phys. Rev. Lett. 111 (2013), 031301, 4 pages, arXiv:1303.3576.

[72] Gielen S., Oriti D., Sindoni L., Homogeneous cosmologies as group field theory condensates, J. High Energy Phys. 2014 (2014), no. 6, 013, 69 pages, arXiv:1311.1238.

[73] Giesel K., Thiemann T., Algebraic quantum gravity (AQG). I. Conceptual setup, Classical Quantum Gravity 24 (2007), 2465-2497, gr-qc/0607099.

[74] Giesel K., Thiemann T., Algebraic quantum gravity (AQG). II. Semiclassical analysis, Classical Quantum Gravity 24 (2007), 2499-2564, gr-qc/0607100.

[75] Girelli F., Livine E.R., Oriti D., Four-dimensional deformed special relativity from group field theories, Phys. Rev. D 81 (2010), 024015, 14 pages, arXiv:0903.3475.

[76] Goroff M.H., Sagnotti A., The ultraviolet behavior of Einstein gravity, Nuclear Phys. B 266 (1986), 709-736.

[77] Guedes C., Oriti D., Raasakka M., Quantization maps, algebra representation, and non-commutative Fourier transform for Lie groups, J. Math. Phys. 54 (2013), 083508, 31 pages, arXiv:1301.7750.

[78] Gurau R., Colored group field theory, Comm. Math. Phys. 304 (2011), 69-93, arXiv:0907.2582.

[79] Gurau R., The 1/N expansion of tensor models beyond perturbation theory, Comm. Math. Phys. 330 (2014), 973-1019, arXiv:1304.2666.

[80] Gurau R., Ryan J.P., Colored tensor models - a review, SIGMA 8 (2012), 020, 78 pages, arXiv:1109.4812.

[81] Han M., Zhang M., Asymptotics of spinfoam amplitude on simplicial manifold: Lorentzian theory, Classical Quantum Gravity 30 (2013), 165012, 57 pages, arXiv:1109.0499.

[82] Henson J., The causal set approach to quantum gravity, in Approaches to Quantum Gravity, Editor D. Oriti, Cambridge University Press, Cambridge, 2009, 393-413, gr-qc/0601121.

[83] Höhn P.A., Quantization of systems with temporally varying discretization. I. Evolving Hilbert spaces, J. Math. Phys. 55 (2014), 083508, 45 pages, arXiv:1401.6062.

[84] Hu B.L., Can spacetime be a condensate?, Internat. J. Theoret. Phys. 44 (2005), 1785-1806, gr-qc/0503067.

[85] Ijjas A., Steinhardt P.J., Loeb A., Inflationary paradigm in trouble after Planck2013, Phys. Lett. B 723 (2013), 261-266, arXiv:1304.2785.

[86] Jacobson T., Thermodynamics of spacetime: the Einstein equation of state, Phys. Rev. Lett. 75 (1995), 1260-1263, gr-qc/9504004.

[87] Kiefer C., Quantum gravity, International Series of Monographs on Physics, Vol. 155, 3rd ed., Oxford University Press, Oxford, 2012.

[88] Kiefer C., Krämer M., Quantum gravitational contributions to the cosmic microwave background anisotropy spectrum, Phys. Rev. Lett. 108 (2012), 021301, 4 pages, arXiv:1103.4967.

[89] Kiefer C., Polarski D., Starobinsky A.A., Quantum-to-classical transition for fluctuations in the early universe, Internat. J. Modern Phys. D 7 (1998), 455-462, gr-qc/9802003.

[90] Koslowski T.A., Dynamical quantum geometry (DQG programme), arXiv:0709.3465.

[91] Krajewski T., Group field theories, PoS Proc. Sci. (2011), PoS(QGQGS2011), 005, 58 pages, arXiv:1210.6257.

[92] Lehners J.-L., Classical inflationary and ekpyrotic universes in the no-boundary wavefunction, Phys. Rev. D 91 (2015), 083525, 18 pages, arXiv:1502.00629.

[93] Lewandowski J., Okołów A., Sahlmann H., Thiemann T., Uniqueness of diffeomorphism invariant states on holonomy-flux algebras, Comm. Math. Phys. 267 (2006), 703-733, gr-qc/0504147. 
[94] Livine E.R., Oriti D., Ryan J.P., Effective Hamiltonian constraint from group field theory, Classical Quantum Gravity 28 (2011), 245010, 17 pages, arXiv:1104.5509.

[95] Maldacena J., The large $N$ limit of superconformal field theories and supergravity, Adv. Theor. Math. Phys. 2 (1998), 231-252, hep-th/9711200.

[96] Mandel L., Wolf E., Optical coherence and quantum optics, Cambridge University Press, Cambridge, 1995.

[97] Markopoulou F., Space does not exist, so time can, arXiv:0909.1861.

[98] Marolf D., Emergent gravity requires kinematic nonlocality, Phys. Rev. Lett. 114 (2015), 031104, 5 pages, arXiv:1409.2509.

[99] Misner C.W., Quantum cosmology. I, Phys. Rev. 186 (1969), 1319-1327.

[100] Ooguri H., Topological lattice models in four dimensions, Modern Phys. Lett. A 7 (1992), 2799-2810, hep-th/9205090.

[101] Oriti D., A quantum field theory of simplicial geometry and the emergence of space-time, J. Phys. Conf. Ser. 67 (2007), 012052, 10 pages, hep-th/0612301.

[102] Oriti D., Group field theory as the microscopic description of the quantum spacetime fluid: a new perspective on the continuum in quantum gravity, PoS Proc. Sci. (2007), PoS(QG-Ph), 030, 38 pages, arXiv:0710.3276.

[103] Oriti D., The microscopic dynamics of quantum space as a group field theory, in Foundations of Space and Time: Reflections on Quantum Gravity, Editors J. Murugan, A. Weltman, G. Ellis, Cambridge University Press, Cambridge, 2012, 257-320, arXiv:1110.5606.

[104] Oriti D., Disappearance and emergence of space and time in quantum gravity, Stud. Hist. Philos. Sci. B Stud. Hist. Philos. Modern Phys. 46 (2014), 186-199, arXiv:1302.2849.

[105] Oriti D., Group field theory as the second quantization of loop quantum gravity, Classical Quantum Gravity 33 (2016), 085005, 32 pages, arXiv:1310.7786.

[106] Oriti D., Pranzetti D., Ryan J.P., Sindoni L., Generalized quantum gravity condensates for homogeneous geometries and cosmology, Classical Quantum Gravity 32 (2015), 235016, 40 pages, arXiv:1501.00936.

[107] Oriti D., Pranzetti D., Sindoni L., Horizon entropy from quantum gravity condensates, Phys. Rev. Lett. 116 (2016), 211301, 6 pages, arXiv:1510.06991.

[108] Oriti D., Ryan J., Group field theory formulation of 3D quantum gravity coupled to matter fields, Classical Quantum Gravity 23 (2006), 6543-6575, gr-qc/0602010.

[109] Oriti D., Ryan J.P., Thürigen J., Group field theories for all loop quantum gravity, New J. Phys. 17 (2015), 023042, 46 pages, arXiv:1409.3150.

[110] Oriti D., Sindoni L., Toward classical geometrodynamics from the group field theory hydrodynamics, New J. Phys. 13 (2011), 025006, 44 pages, arXiv:1010.5149.

[111] Oriti D., Sindoni L., Wilson-Ewing E., Emergent Friedmann dynamics with a quantum bounce from quantum gravity condensates, arXiv:1602.05881.

[112] Oriti D., Sindoni L., Wilson-Ewing E., Bouncing cosmologies from quantum gravity condensates, arXiv:1602.08271.

[113] Ousmane Samary D., Closed equations of the two-point functions for tensorial group field theory, Classical Quantum Gravity 31 (2014), 185005, 29 pages, arXiv:1401.2096.

[114] Pachner U., P.L. homeomorphic manifolds are equivalent by elementary shellings, European J. Combin. 12 (1991), 129-145.

[115] Padmanabhan T., Emergent gravity paradigm: recent progress, Modern Phys. Lett. A 30 (2015), 1540007, 21 pages, arXiv:1410.6285.

[116] Peldán P., Actions for gravity, with generalizations: a review, Classical Quantum Gravity 11 (1994), 1087-1132, gr-qc/9305011.

[117] Perez A., The spin foam approach to quantum gravity, Living Rev. Relativ. 16 (2013), 3, 128 pages, arXiv:1205.2019.

[118] Pitaevskii L., Stringari S., Bose-Einstein condensation, International Series of Monographs on Physics, Vol. 116, The Clarendon Press, Oxford University Press, Oxford, 2003.

[119] Pithis A.G.A., Sakellariadou M., Tomov P., Impact of nonlinear effective interactions on GFT quantum gravity condensates, arXiv:1607.06662. 
[120] Ponzano G., Regge T., Semiclassical limit of Racah coefficients, in Spectroscopy and Group Theoretical Methods in Physics, Editor F. Block, North Holland, Amsterdam, 1968, 1-58.

[121] Reisenberger M.P., Rovelli C., Spacetime as a Feynman diagram: the connection formulation, Classical Quantum Gravity 18 (2001), 121-140, gr-qc/0002095.

[122] Rivasseau V., The tensor track, III, Fortschr. Phys. 62 (2014), 81-107, arXiv:1311.1461.

[123] Rivasseau V., Why are tensor field theories asymptotically free?, Europhys. Lett. 111 (2015), 60011, 6 pages, arXiv:1507.04190.

[124] Rovelli C., Quantum gravity, Cambridge Monographs on Mathematical Physics, Cambridge University Press, Cambridge, 2004.

[125] Rovelli C., Zakopane lectures on loop gravity, PoS Proc. Sci. (2011), PoS(QGQGS2011), 003,60 pages, arXiv:1102.3660.

[126] Rovelli C., Smolin L., Discreteness of area and volume in quantum gravity, Nuclear Phys. B 442 (1995), 593-619, Erratum, Nuclear Phys. B 456 (1995), 753-754, gr-qc/9411005.

[127] Sahlmann H., On loop quantum gravity kinematics with a non-degenerate spatial background, Classical Quantum Gravity 27 (2010), 225007, 17 pages, arXiv:1006.0388.

[128] Sindoni L., Emergent models for gravity: an overview of microscopic models, SIGMA 8 (2012), 027, 45 pages, arXiv:1110.0686.

[129] Sindoni L., Effective equations for GFT condensates from fidelity, arXiv:1408.3095.

[130] Sorkin R.D., Spacetime and causal sets, in Relativity and Gravitation: Classical and Quantum (Cocoyoc, 1990), World Sci. Publ., River Edge, NJ, 1991, 150-173.

[131] Thiemann T., Modern canonical quantum general relativity, Cambridge Monographs on Mathematical Physics, Cambridge University Press, Cambridge, 2007, gr-qc/0110034.

[132] Unruh W.G., Experimental black-hole evaporation?, Phys. Rev. Lett. 46 (1981), 1351-1353.

[133] Wands D., Malik K.A., Lyth D.H., Liddle A.R., New approach to the evolution of cosmological perturbations on large scales, Phys. Rev. D 62 (2000), 043527, 8 pages, astro-ph/0003278.

[134] Wiltshire D.L., An introduction to quantum cosmology, in Proceedings of 8th Physics Summer School on Cosmology: The Physics of the Universe (Canberra, 1995), Editors B. Robson, N. Visvanathan, W.S. Woolcock, World Sci., Singapore, 1996, 473-531, gr-qc/0101003.

[135] Zanardi P., Paunković N., Ground state overlap and quantum phase transitions, Phys. Rev. E 74 (2006), 031123, 6 pages, quant-ph/0512249. 